\title{
DRIFT EFFECTS AND THE COSMIC RAY DENSITY GRADIENT IN A SOLAR ROTATION PERIOD: FIRST OBSERVATION WITH THE GLOBAL MUON DETECTOR NETWORK (GMDN)
}

\author{
Y. Okazaki, ${ }^{1}$ A. Fushishita, ${ }^{2}$ T. Narumi, ${ }^{2}$ C. Kato, ${ }^{2}$ S. Yasue, ${ }^{2}$ T. Kuwabara, ${ }^{3}$ J. W. Bieber ${ }^{3}$ \\ P. Evenson, ${ }^{3}$ M. R. Da Silva, ${ }^{4}$ A. Dal Lago, ${ }^{4}$ N. J. Schuch, ${ }^{5}$ Z. FujiI, ${ }^{6}$ M. L. Duldig, ${ }^{7}$ \\ J. E. Humble, ${ }^{8}$ I. Sabbah, ${ }^{9}$ J. Kóta, ${ }^{10}$ and K. Munakata ${ }^{2}$ \\ Received 2007 December 26; accepted 2008 March 17
}

\begin{abstract}
We present for the first time hourly variations of the spatial density gradient of $50 \mathrm{GeV}$ cosmic rays within a sample solar rotation period in 2006. By inversely solving the diffusive flux equation, including the drift, we deduce the gradient from the anisotropy that is derived from the observation made by the Global Muon Detector Network (GMDN). The anisotropy obtained by applying a new analysis method to the GMDN data is precise and free from atmospheric temperature effects on the muon count rate recorded by ground-based detectors. We find the derived north-south gradient perpendicular to the ecliptic plane is oriented toward the heliospheric current sheet (HCS; i.e., southward in the toward sector of the interplanetary magnetic field [IMF] and northward in the away sector). The orientation of the gradient component parallel to the ecliptic plane remains similar in both sectors, with an enhancement of its magnitude seen after the Earth crosses the HCS. These temporal features are interpreted in terms of a local maximum of the cosmic ray density at the HCS. This is consistent with the prediction of the drift model for the $A<0$ epoch. By comparing the observed gradient with the numerical prediction of a simple drift model, we conclude that particle drifts in the large-scale magnetic field play an important role in organizing the density gradient, at least in the present $A<0$ epoch. We also found that corotating interaction regions did not have such a notable effect. Observations with the GMDN provide us with a new tool for investigating cosmic-ray transport in the IMF.
\end{abstract}

Subject headings: cosmic rays — solar wind - Sun: magnetic fields

\section{INTRODUCTION}

The drift model of cosmic ray transport in the heliosphere predicts a bidirectional latitude gradient of the galactic cosmic ray (GCR) density, pointing in opposite directions on opposite sides of the heliospheric current sheet (HCS) (Jokipii \& Kóta 1982). The predicted spatial distribution of the GCR density has a minimum along the HCS in the "positive" polarity period of the solar polar magnetic field (also referred as the $A>0$ epoch), when the interplanetary magnetic field (IMF) directs away from (toward) the Sun in the northern (southern) hemisphere, while the distribution has the local maximum on the HCS in the "negative" period $(A<0$ epoch) with the opposite field orientation in each hemisphere. The field orientation reverses every 11 years around the maximum period of solar activity. By analyzing the GCR

\footnotetext{
${ }^{1}$ Department of Geophysics, Tohoku University, Sendai, Miyagi 980-0861, Japan; okazaki@pat.geophys.tohoku.ac.jp.

2 Physics Department, Shinshu University, Matsumoto, Nagano 390-8621, Japan; kmuna00@gipac.shinshu-u.ac.jp.

3 Bartol Research Institute and Department of Physics and Astronomy, University of Delaware, Newark, DE 19716; jwbieber@bartol.udel.edu.

4 National Institute for Space Research (INPE), 12227-010 São Jose dos Campos, SP, Brazil; dallago@dge.inpe.br.

5 Southern Regional Space Research Center (CRS/INPE), P.O. Box 5021, 97110-970, Santa Maria, RS, Brazil; njschuch@lacesm.ufsm.br.

6 Solar Terrestrial Environment Laboratory, Nagoya University, Nagoya, Aichi 464-8601, Japan; fujii@stelab.nagoya-u.ac.jp.

7 Australian Antarctic Division, Kingston, Tasmania 7050, Australia; marc .duldig@aad.gov.au.

8 School of Mathematics and Physics, University of Tasmania, Hobart, Tasmania 7001, Australia; john.humble@utas.edu.au.

9 Department of Physics, Faculty of Science, Kuwait University, Kuwait; on leave from Physics Department, Faculty of Science, University of Alexandria, Egypt; sabbahsom@yahoo.com.

10 Lunar and Planetary Laboratory, University of Arizona, Tucson, AZ 87721; kota@LPL.Arizona.EDU.
}

density measured with the anticoincidence guard on board $A C E$ and Helios satellites and by ground-based neutron monitors (NMs), Richardson et al. (1996) and Richardson (2004) investigated GCR modulation by corotating interaction regions (CIRs), which are formed at the leading edges of corotating high-speed solar wind streams originating in coronal holes on the solar surface. The anticoincidence guards measure sub-GeV GCRs, while the NMs have a median response to primary particles with energies of 10-20 GeV. These analyses were based on a onedimensional spatial distribution of the GCR density measured as the temporal variation of the GCR count rate recorded by each detector as it sweeps across the CIR. From statistical analyses of variations in many CIRs with and without the HCS, the above authors concluded that the IMF sector boundary (i.e., HCS) does not organize the GCR density, contrary to expectations from the simple drift model. On the other hand, they also found observational evidence that the amplitude of the CIR-related modulations of the GCR density around the solar minimum is $\sim 50 \%$ larger in $A>0$ epochs than in $A<0$ epochs. The simple drift model also predicts different modulation amplitudes in $A>0$ and $A<0$ epochs. Despite opposite latitudinal gradients, the longitudinal profile has the same sense; the intensity being higher at the sector crossing and lower away from the current sheet in both cases (Jokipii \& Kóta 1982). The magnitude of the drift effect on the GCR modulation in CIRs is still an open question.

The spatial distribution of the GCR density can also be inferred from measuring the anisotropic streaming of GCRs at the Earth, as the diffusion streaming of GCRs reflects the local spatial gradient of the GCR density. The anisotropic streaming of GCRs in three dimensions manifests itself in the diurnal and "north-south" (NS) anisotropies of the intensity recorded by ground-based detectors. The diurnal anisotropy is the anisotropy component along the equatorial plane, while the NS anisotropy is the component along 
the Earth's spin axis. By analyzing the yearly mean anisotropy observed with several NMs and muon detectors, a series of studies revealed long-term variations of the radial and latitudinal gradients of GCR density in the heliosphere as well as variations in mean free paths of GCRs scattered by magnetic irregularities (Swinson 1969; Bieber \& Chen 1991; Chen \& Bieber 1993; Hall et al. 1996, 1997; Ahluwalia \& Dorman 1997; Sabbah 1999; Munakata et al. 2002). These studies provide important information on the large-scale distribution of GCRs and enable us to make crucial tests of physical models for the modulation of GCRs in the heliosphere. By analyzing the diurnal anisotropy observed with several NMs, Bieber \& Chen (1991) first confirmed the existence of the bidirectional latitude gradient predicted by the drift model. The gradient reversed with the solar magnetic polarity reversal at approximately every solar sunspot maximum. Similar results were also reported from observations with muon detectors monitoring the GCR intensity at higher energies (Hall et al. 1996, 1997; Munakata et al. 2002).

These studies, however, only reported the yearly mean diurnal anisotropy. Bieber \& Chen (1991) actually analyzed the average anisotropy over the 27 day solar rotation period, but used 14 rotation averages in a year for evaluating the error of the yearly mean. No information was deduced about the temporal evolution of the density gradient over shorter periods within each solar rotation, such as evolutions on day-to-day or hourly timescales. The diurnal variation of GCR intensity recorded in a single ground-based detector gives the correct anisotropy only when the anisotropy is stationary at least for one day, during which the detector scans $360^{\circ}$ longitude in space. In reality, the anisotropic streaming in three dimensions can change dynamically in both magnitude and orientation. Accurate analysis of such dynamic changes requires continuous monitoring of the GCR intensity over the entire sky. Therefore, it is important to differentiate the temporal variation of the anisotropy from the variation of the omnidirectional intensity (i.e., the GCR density). Such monitoring has been made possible by construction of the worldwide networks of NMs and muon detectors (Bieber \& Evenson 1998; Kuwabara et al. 2004).

In this paper, we study the GCR modulation in CIRs for the first time by analyzing the spatial gradient of the high-energy GCR density in three dimensions. The gradient gives information on the remote distribution surrounding the detector view. We derive the density gradient from the GCR anisotropy observed with the Global Muon Detector Network (GMDN), which began operation in March 2006. The GMDN has been designed and constructed to precisely measure the anisotropy. The responses of different surface muon detectors to GCRs have median energies ranging from 50 to $150 \mathrm{GeV}$. These high-energy GCRs have large Larmor radii in the IMF $(\sim 0.2 \mathrm{AU}$ at $5 \mathrm{nT})$ and are less sensitive to small-scale irregular structures in the solar wind. Observations made by the GMDN are therefore expected to provide us with a new tool for investigating the large-scale structure in CIRs. Specifically, we develop a new analysis method by which we can eliminate the atmospheric temperature effect on the muon count rate and precisely examine the temporal evolution of the NS anisotropy, even over a period shorter than a day. By applying this method to data observed during CIRs in 2006 and 2007, we demonstrate that combining the GMDN and the new analysis method enable us to monitor dynamic variations of the anisotropy in association with sector boundary crossings by the Earth. We then compare the derived density gradient with the prediction of the drift model.

The outline of this paper is as follows. In $\S 2$ we briefly describe the GMDN. In $\S 3$, we derive the spatial gradient of the cosmic-ray density from the anisotropy in three dimensions, which is obtained by best-fitting to the data observed with the GMDN, and compare the derived density gradient with numerical results obtained from a simple drift model. Our conclusions and discussions are given in $\S 4$. A new analysis method, which is employed for obtaining the anisotropy free from the atmospheric temperature effect, is developed and described in Appendix A. A separate analysis of the temperature effect using the high-altitude atmospheric data is also given in Appendix B. This proves that the temperature effect was successfully eliminated by the new analysis method.

\section{GLOBAL MUON DETECTOR NETWORK (GMDN)}

Data acquisition by the network began in December 1992, as two-hemisphere observations using a pair of muon detectors at Nagoya (Japan) and Hobart (Australia), which have detection areas of $36 \mathrm{~m}^{2}$ and $9 \mathrm{~m}^{2}$, respectively. Each of these detectors is multidirectional, allowing us to simultaneously record the intensities in various directions of viewing. Another small $\left(4 \mathrm{~m}^{2}\right)$ detector, prototype São Martinho (Brazil), was added to the network in March 2001 to fill a gap in directional coverage of the network over the Atlantic and Europe. These detectors have identical design, except for their detection area, consisting of two horizontal layers of plastic scintillators, vertically separated by $1.73 \mathrm{~m}$, with an intermediate $5 \mathrm{~cm}$ layer of lead to absorb the soft component. Each layer comprises an array of $1 \mathrm{~m}^{2}$ unit detectors, each with a $1 \mathrm{~m} \times 1 \mathrm{~m}$ plastic scintillator viewed by a photomultiplier tube of $12.7 \mathrm{~cm}$ diameter. By counting pulses of the twofold coincidences between a pair of detectors on the upper and lower layers, we can record the rate of muons from the corresponding incident direction. The multidirectional muon telescope comprises various combinations between the upper and lower detectors. The prototype São Martinho was upgraded in December 2005 by expanding its detection area to $28 \mathrm{~m}^{2}$.

In March 2006, the Global Muon Detector Network (GMDN) was finally completed by installing a new detector at Kuwait University (Kuwait), with a detection area of $9 \mathrm{~m}^{2}$. This adds new directions of viewing over the African Continent and the western Indian Ocean. The Kuwait University muon detector is a hodoscope designed specifically for measuring the "loss cone" anisotropy, which is observed as a precursor to the arrival of interplanetary shocks at Earth and is characterized by an intensity deficit confined to a narrow pitch angle region around the sunward IMF direction (Munakata et al. 2000; Leerungnavarat et al. 2003). Unlike the other three detectors, the Kuwait University detector consists of four horizontal layers of 30 proportional counter tubes (PCTs). Each PCT is a $5 \mathrm{~m}$ long cylinder with a $10 \mathrm{~cm}$ diameter having a $50 \mu \mathrm{m}$ thick tungsten anode along the cylinder axis. A $5 \mathrm{~cm}$ layer of lead is installed above the detector to absorb the soft component. The PCT axes are aligned geographic east-west $(X)$ in the second and bottom layers and north-south $(Y)$ in the top and third layers. The top and second layers form an upper pair, while the third and bottom layers form a lower pair. The two pairs are separated vertically by $80 \mathrm{~cm}$. Muon recording is triggered by the fourfold coincidence of pulses from all layers and the incident direction is identified from $X-Y$ locations of the upper and lower PCT pairs. This is approximately equivalent to recording muons with two $30 \times 30$ square arrays of $10 \mathrm{~cm} \times 10 \mathrm{~cm}$ detectors separated vertically by $80 \mathrm{~cm}$. The muon count is recorded in each of $23 \times 23=529$ directional channels which cover $360^{\circ}$ of azimuth angle and $0^{\circ}$ to $60^{\circ}$ zenith. For analyzing Kuwait University data together with the data from the other three detectors of different geometry, we convert 529 directional channels into 13 channels, which are equivalent to those in Hobart having the same detection area $\left(9 \mathrm{~m}^{2}\right)$. For the performance 
TABLE 1

Characteristics of the Global Muon Detector Network

\begin{tabular}{|c|c|c|c|c|c|c|c|}
\hline $\begin{array}{c}\text { Directional } \\
\text { Channel }\end{array}$ & $\begin{array}{l}\text { Count Rate } \\
\left(10^{4} \mathrm{hr}^{-1}\right)\end{array}$ & $\begin{array}{c}\sigma_{c} \\
(\%)\end{array}$ & $\begin{array}{c}P_{m} \\
(\mathrm{GV})\end{array}$ & $\begin{array}{l}\lambda_{\text {asymp }} \\
(\text { deg) }\end{array}$ & $\begin{array}{c}\phi_{\text {asymp }} \\
(\mathrm{deg})\end{array}$ & $\gamma_{\text {temp }}$ & $\begin{array}{c}\beta_{\text {temp }} \\
\left(\% \mathrm{~km}^{-1}\right)\end{array}$ \\
\hline \multicolumn{8}{|c|}{ Nagoya $\left(35.1^{\circ} \mathrm{N}, 137.0^{\circ} \mathrm{E}, 77 \mathrm{~m}\right)$} \\
\hline V ……............... & 276 & 0.06 & 59.4 & 28.0 & 168.4 & -0.947 & -6.83 \\
\hline $\mathrm{N}$ & 125 & 0.09 & 64.6 & 47.0 & 192.7 & -0.950 & -7.11 \\
\hline $\mathrm{S} \ldots \ldots \ldots \ldots \ldots \ldots$ & 123 & 0.10 & 62.6 & 2.9 & 157.5 & -0.948 & -7.10 \\
\hline $\mathrm{E} \ldots \ldots \ldots \ldots \ldots \ldots \ldots \ldots$ & 120 & 0.09 & 66.7 & 10.8 & 194.0 & -0.950 & -7.08 \\
\hline $\mathrm{W} \ldots \ldots \ldots \ldots \ldots$ & 126 & 0.09 & 61.8 & 40.2 & 135.0 & -0.949 & -7.16 \\
\hline 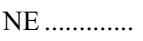 & 58 & 0.14 & 72.0 & 25.9 & 209.4 & -0.953 & -7.29 \\
\hline NW ................. & 62 & 0.13 & 66.6 & 64.3 & 156.4 & -0.952 & -7.37 \\
\hline SE .................. & 58 & 0.14 & 69.3 & -6.6 & 182.4 & -0.953 & -7.37 \\
\hline SW................. & 60 & 0.13 & 65.6 & 12.8 & 131.1 & -0.949 & -7.35 \\
\hline $\mathrm{N} 2 \ldots \ldots \ldots \ldots$ & 61 & 0.13 & 83.0 & 56.1 & 217.0 & -0.960 & -7.66 \\
\hline $\mathrm{S} 2 .$. & 60 & 0.13 & 80.5 & -14.1 & 152.2 & -0.958 & -7.60 \\
\hline E2 ................... & 58 & 0.14 & 88.3 & 2.0 & 206.8 & -0.959 & -7.51 \\
\hline W2 .................. & 62 & 0.13 & 79.3 & 40.4 & 105.0 & -0.958 & -7.54 \\
\hline $\mathrm{N} 3 \ldots \ldots \ldots \ldots$ & 18 & 0.27 & 105.0 & 59.5 & 236.1 & -0.945 & -7.77 \\
\hline S3 ................... & 18 & 0.27 & 103.7 & -24.4 & 149.6 & -0.941 & -7.79 \\
\hline & 17 & 0.28 & 113.7 & -1.7 & 213.4 & -0.938 & -7.61 \\
\hline W3 ................. & 18 & 0.27 & 103.0 & 35.6 & 87.5 & -0.945 & -7.59 \\
\hline
\end{tabular}

Hobart $\left(43.0^{\circ} \mathrm{S}, 147.3^{\circ} \mathrm{E}, 65 \mathrm{~m}\right)$

\begin{tabular}{|c|c|c|c|c|c|c|c|}
\hline V ……............. & 83 & 0.12 & 54.6 & -40.0 & 170.5 & -0.773 & -4.44 \\
\hline $\mathrm{N}$ & 29 & 0.20 & 59.0 & -17.5 & 152.5 & -0.767 & -4.47 \\
\hline S _.................. & 30 & 0.20 & 59.0 & -53.9 & 206.0 & -0.783 & -4.62 \\
\hline E .................... & 30 & 0.20 & 59.0 & -18.6 & 193.5 & -0.749 & -4.57 \\
\hline W.................... & 29 & 0.20 & 59.0 & -55.6 & 132.4 & -0.770 & -4.51 \\
\hline NE .................. & 12 & 0.33 & 63.7 & -3.9 & 176.0 & -0.696 & -4.46 \\
\hline NW .... & 11 & 0.33 & 63.7 & -29.1 & 125.8 & -0.716 & -4.31 \\
\hline SE .................. & 12 & 0.33 & 63.7 & -30.2 & 214.8 & -0.696 & -4.31 \\
\hline 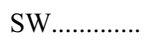 & 12 & 0.33 & 63.7 & -77.2 & 171.6 & -0.756 & -4.54 \\
\hline $\mathrm{N} 2 \ldots \ldots \ldots \ldots$ & 7.0 & 0.42 & 76.3 & 0.2 & 145.6 & -0.695 & -4.88 \\
\hline S2 ................ & 7.3 & 0.42 & 76.3 & -57.6 & 236.7 & -0.698 & -4.64 \\
\hline E2 ................... & 7.2 & 0.42 & 76.3 & -6.4 & 205.8 & -0.649 & -4.39 \\
\hline W2 .................. & 7.1 & 0.42 & 76.3 & -53.2 & 95.6 & -0.735 & -7.56 \\
\hline
\end{tabular}

São Martinho $\left(29.4^{\circ} \mathrm{S}, 306.2^{\circ} \mathrm{E}, 488 \mathrm{~m}\right)$

\begin{tabular}{|c|c|c|c|c|c|c|c|}
\hline V..... & 231 & 0.07 & 55.6 & -22.6 & 330.4 & -0.735 & -4.18 \\
\hline $\mathrm{N}$ & 88 & 0.11 & 59.8 & 5.3 & 325.3 & -0.680 & -3.83 \\
\hline & 91 & 0.11 & 59.1 & -48.3 & 347.2 & -0.734 & -4.37 \\
\hline ........ & 102 & 0.10 & 61.7 & -10.7 & 358.5 & -0.711 & -3.96 \\
\hline W ..................... & 102 & 0.10 & 58.3 & -29.1 & 298.0 & -0.713 & -4.02 \\
\hline NE ................. & 42 & 0.15 & 66.6 & 10.3 & 350.3 & -0.665 & -3.67 \\
\hline NW ................. & 42 & 0.15 & 62.7 & -0.9 & 299.0 & -0.653 & -3.71 \\
\hline SE ............ & 43 & 0.15 & 65.2 & -30.6 & 11.2 & -0.688 & -4.05 \\
\hline SW.. & 43 & 0.15 & 62.3 & -56.8 & 304.0 & -0.710 & -4.01 \\
\hline $\mathrm{N} 2 \ldots \ldots \ldots \ldots$ & 29 & 0.17 & 79.0 & 23.0 & 322.5 & -0.636 & -3.45 \\
\hline S2 .................. & 30 & 0.17 & 77.3 & -63.1 & 8.8 & -0.692 & -4.09 \\
\hline E2 …............... & 37 & 0.15 & 80.6 & -3.6 & 12.9 & -0.638 & -3.66 \\
\hline W2 .................. & 37 & 0.15 & 75.0 & -27.7 & 273.0 & -0.707 & -4.01 \\
\hline N3 ................... & 3.4 & 0.46 & 99.0 & 33.3 & 321.5 & -0.494 & -3.69 \\
\hline S3 ……........... & 3.5 & 0.46 & 96.9 & -68.6 & 32.6 & -0.589 & -5.11 \\
\hline E3 ……............ & 7.5 & 0.30 & 105.0 & -0.7 & 20.0 & -0.637 & -4.58 \\
\hline W3 .................. & 7.7 & 0.30 & 98.8 & -23.7 & 257.9 & -0.654 & -4.39 \\
\hline
\end{tabular}

Kuwait University ${ }^{\mathrm{a}}\left(29.3^{\circ} \mathrm{N}, 48.0^{\circ} \mathrm{E}, 0 \mathrm{~m}\right)$

\begin{tabular}{|c|c|c|c|c|c|c|c|}
\hline V.. & 86 & 0.10 & 62.3 & 24.2 & 77.2 & -0.945 & -6.43 \\
\hline N.. & 22 & 0.19 & 67.8 & 61.4 & 76.3 & -0.948 & -6.55 \\
\hline ......... & 22 & 0.19 & 69.4 & -11.9 & 90.1 & -0.948 & -6.85 \\
\hline E & 22 & 0.20 & 73.5 & 18.1 & 121.1 & -0.950 & -6.36 \\
\hline W..................... & 22 & 0.19 & 66.0 & 12.6 & 36.4 & -0.945 & -6.73 \\
\hline NE ................. & 6.4 & 0.35 & 78.2 & 44.4 & 127.9 & -0.946 & -6.55 \\
\hline NW ................... & 6.5 & 0.34 & 72.9 & 45.1 & 18.8 & -0.942 & -6.50 \\
\hline SE .................... & 6.5 & 0.35 & 82.3 & -6.0 & 119.7 & -0.946 & -6.35 \\
\hline
\end{tabular}

TABLE 1 -Continued

\begin{tabular}{|c|c|c|c|c|c|c|c|}
\hline $\begin{array}{c}\text { Directional } \\
\text { Channel }\end{array}$ & $\begin{array}{l}\text { Count Rate } \\
\left(10^{4} \mathrm{hr}^{-1}\right)\end{array}$ & $\begin{array}{c}\sigma_{c} \\
(\%)\end{array}$ & $\begin{array}{l}P_{m} \\
(\mathrm{GV})\end{array}$ & $\begin{array}{c}\lambda_{\text {asymp }} \\
(\text { deg) }\end{array}$ & $\begin{array}{c}\phi_{\text {asymp }} \\
(\text { deg) }\end{array}$ & $\gamma_{\text {temp }}$ & $\begin{array}{c}\beta_{\text {temp }} \\
\left(\% \mathrm{~km}^{-1}\right)\end{array}$ \\
\hline \multicolumn{8}{|c|}{ Kuwait University $^{\mathrm{a}}\left(29.3^{\circ} \mathrm{N}, 48.0^{\circ} \mathrm{E}, 0 \mathrm{~m}\right)$} \\
\hline 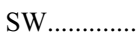 & 6.8 & 0.34 & 73.4 & -17.4 & 56.1 & -0.939 & -7.10 \\
\hline $\mathrm{N} 2 \ldots$. & 2.8 & 0.47 & 97.9 & 81.9 & 58.9 & -0.916 & -6.14 \\
\hline $\mathrm{S} 2 \ldots \ldots \ldots \ldots$ & 2.8 & 0.47 & 102.2 & -26.1 & 95.9 & -0.908 & -5.94 \\
\hline $\mathrm{E} 2 \ldots \ldots \ldots \ldots . . . .$. & 2.6 & 0.49 & 109.8 & 15.9 & 135.9 & -0.908 & -6.38 \\
\hline W2 .................. & 2.8 & 0.47 & 97.0 & 0.8 & 15.8 & -0.927 & -6.75 \\
\hline
\end{tabular}

Notes.-Average hourly count rate, count rate error $\left(\sigma_{c}\right)$, median primary rigidity $\left(P_{m}\right)$, latitude $\left(\lambda_{\text {asymp }}\right)$ and longitude $\left(\phi_{\text {asymp }}\right)$, correlation $\left(\gamma_{\text {temp }}\right)$ and regression $\left(\beta_{\text {temp }}\right)$ coefficients of the correlation between the $100 \mathrm{hPa}$ altitude and the muon rate are listed for each GMDN directional channel. See Appendix A for $\gamma_{\text {temp }}$ and $\beta_{\text {temp }}$.

${ }^{\text {a }}$ The Kuwait University muon detector uses proportional counters; the other detectors use plastic scintillation counters (see text).

of a muon hodoscope of similar design, readers can refer to Munakata et al. (2005).

Table 1 summarizes the locations of detectors and characteristics of directional telescopes in the GMDN. The total number of directional channels available in the network was 60 at March 2006. The median rigidity $\left(P_{m}\right)$ of primary cosmic rays recorded, calculated by utilizing the response function of the atmospheric muons to the primary particles (Murakami et al. 1979), ranges from 55 to $114 \mathrm{GV}$, and the statistical error $\left(\sigma_{c}\right)$ of hourly count rate ranges between $0.06 \%$ and $0.49 \%$. Each symbol in Figure 1 shows the asymptotic viewing direction, after correction for geomagnetic bending, at rigidity $P_{m}$ of each directional channel, as determined using a particle trajectory code (Lin et al. 1995). The track through each symbol represents the spread of viewing directions for particle rigidities between $P_{0.1}$ and $P_{0.9}$ that confine the central $80 \%$ of each directional channel's energy response. Figure 1 illustrates that the GMDN covers almost the entire sky, although it still has gaps remaining in its directional coverage over North America and the southern Indian Ocean.

\section{GCR MODULATION IN A CIR AND A COMPARISON WITH THE DRIFT MODEL}

In this section, we derive the spatial gradient of the GCR density from the best-fit anisotropy. The analysis method for deriving the GCR anisotropy from the GMDN data is described in detail in Appendix A. We analyze pressure-corrected hourly muon count rates recorded in the 60 directional channels in the GMDN between 2006 March 14 and 2007 March 4. This period covers 13 solar rotations (Carrington rotations CR 2041-2053), during which clear recurrent signatures of CIRs were seen in solar wind parameters measured by the $A C E$ satellite. We chose this period because $A C E$-Level2 data were available. ${ }^{11}$ Aiming to examine the global drift effect on the GCR modulation within a solar rotation period, we now concentrate on analyzing a sample solar rotation (CR 2043). Statistical analyses of multiple rotations will be presented in a separate paper.

\subsection{Derivation of the GCR Density Gradient from the Anisotropy}

We first transform the anisotropy components, $\xi_{x}^{\mathrm{GEO}}(t), \xi_{y}^{\mathrm{GEO}}(t)$, and $\xi_{z}^{\mathrm{GEO}}(t)$ obtained in Appendix A, to the geocentric solar ecliptic (GSE) coordinate system. The coordinate system used hereafter in $\S \S 3$ and 4 is GSE, unless otherwise noted. We then correct the transformed anisotropy vector $\xi^{\mathrm{GSE}}(t)$ for the solar

${ }^{11} A C E$-Level2 data are available at http://www.srl.caltech.edu/ACE/ASC/. 


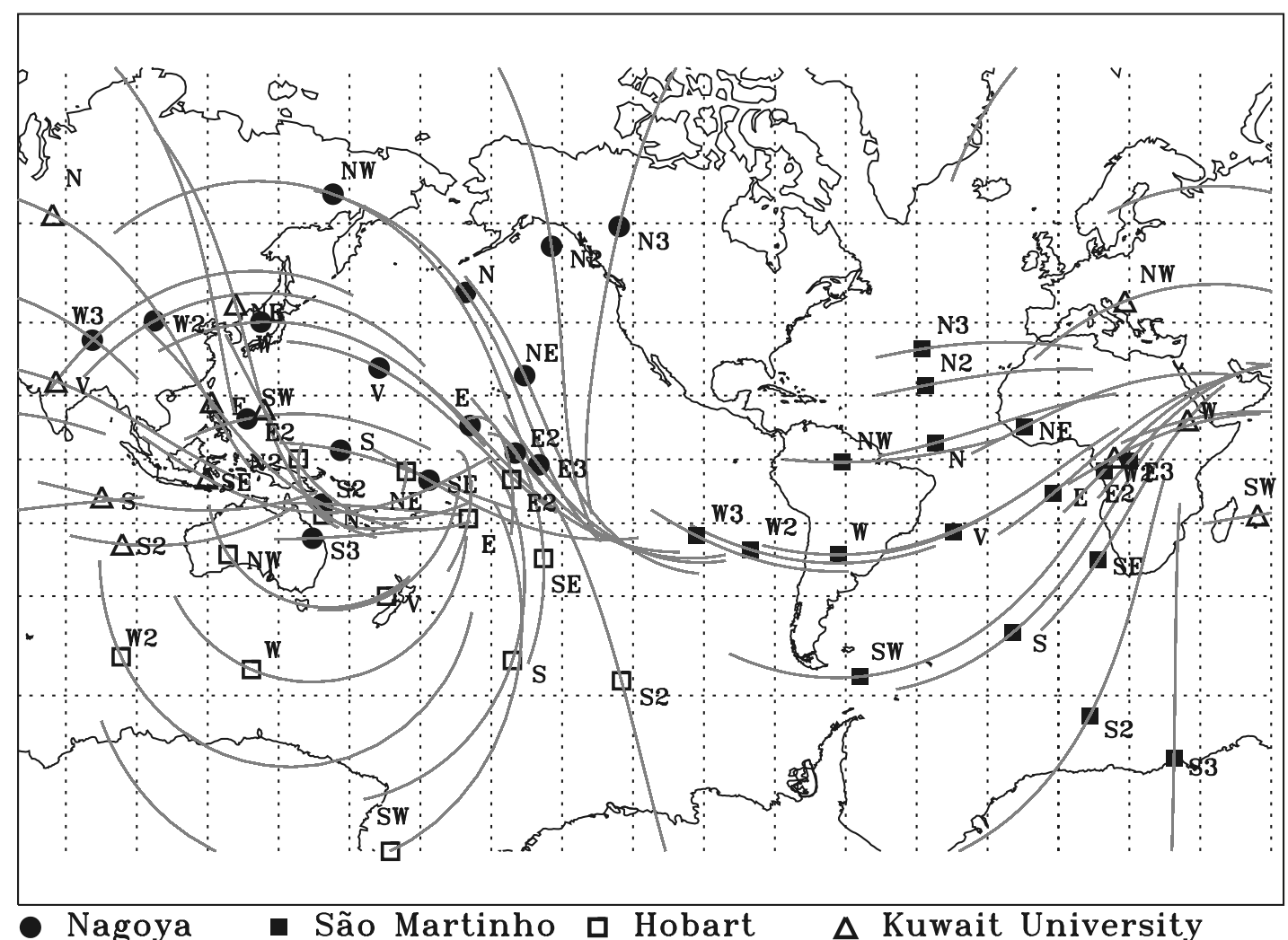

FIG. 1.-Asymptotic viewing directions of the GMDN. Each symbol (filled circles for Nagoya, filled squares for São Martinho, open squares for Hobart, open triangles for Kuwait University) shows the asymptotic viewing direction (after correction for geomagnetic bending) of each directional channel with median primary rigidity $\left(P_{m}\right)$ as listed in Table $1\left(\lambda_{\text {asymp }}, \phi_{\text {asymp }}\right)$. The track through each symbol represents the spread of viewing directions corresponding to the central $80 \%$ of each channel's energy response (see text).

wind convection anisotropy and the Compton-Getting anisotropy arising from the Earth's $30 \mathrm{~km} \mathrm{~s}^{-1}$ orbital motion and obtain the diffusive anisotropy $\boldsymbol{\xi}(t)$ as

$$
\boldsymbol{\xi}(t)=\boldsymbol{\xi}^{\mathrm{GSE}}(t)+(2+\gamma)\left[\boldsymbol{V}_{\mathrm{SW}}(t)-\boldsymbol{v}_{\mathrm{E}}\right] / c,
$$

where $\gamma$ is the power-law index of the GCR energy spectrum, which we set to $2.7, V_{\mathrm{SW}}(t)$ is the solar wind velocity in the $A C E$ Level 2 data, $\boldsymbol{v}_{\mathrm{E}}$ is the velocity of the Earth's orbital motion, and $c$ is the speed of light. For the analysis in this paper, we use the $A C E$ IMF and solar wind data lagged by one hour, as a rough correction for the solar wind transit time between $A C E$ and the Earth.

We define the IMF sector polarity based on the hourly mean IMF components in the GSE in $A C E$-Level2 data. As the spiral angle of the IMF measured near the Earth is, on average, about $45^{\circ}$, the toward (the Sun) and away (from the Sun) sectors are defined relative to a plane normal to the $45^{\circ}$ spiral in the ecliptic plane; i.e., in GSE coordinates the IMF is designated toward (T) if $B_{x}>B_{y}$ and away (A) if $B_{y}>B_{x}$.

Figures $2 b-2 d$ show hourly values of the three GSE components of $\boldsymbol{\xi}(t)$ in CR 2043, while Figure $2 a$ shows the best-fit density $I_{0}(t)$, each as a function of time $t$ in the day of year [DOY; see Appendix A for $\left.I_{0}(t)\right]$. The identified times of the Earth's HCS crossings are indicated by vertical solid lines, while the polarity ( T/A) of each IMF sector between the HCS crossings is indicated at the top of the figure. The dispersion of each parameter due to the counting error $\left(\sigma_{c}\right.$ in Table 1$)$ is evaluated on the basis of the numerical simulation of the best-fit to be $0.06 \%$ for $I_{0}(t), 0.05 \%$ for $\xi_{x}(t), 0.06 \%$ for $\xi_{y}(t)$, and $0.07 \%$ for $\xi_{z}(t)$. Note that in Figure $2 d \xi_{z}(t)$ is positive in the T sectors for the majority of hours, while it is negative in the A sectors. Such a feature is much harder to see in the hourly values of the GG component in Figure $2 e$, which has long been used as a measure of the north-south anisotropy, due to statistical fluctuations (see Appendix A for the GG component). It is also noted that $\xi_{x}(t)$ and $\xi_{y}(t)$ in Figures $2 b$ and $2 c$ remain respectively negative and positive in most hours, regardless of the sector polarity. We derive the GCR density gradient in three dimensions from $\boldsymbol{\xi}(t)$ in this figure.

We divide the observed anisotropy vector $\boldsymbol{\xi}(t)$ into components parallel and perpendicular to the IMF as

$$
\boldsymbol{\xi}(t)=\boldsymbol{\xi}_{\|}(t)+\boldsymbol{\xi}_{\perp}(t) .
$$

These components are given as

$$
\begin{gathered}
\boldsymbol{\xi}_{\|}(t)=R_{\mathrm{L}}(t) \alpha_{\|} \boldsymbol{G}_{\|}(t), \\
\boldsymbol{\xi}_{\perp}(t)=R_{\mathrm{L}}(t)\left[\alpha_{\perp} \boldsymbol{G}_{\perp}(t)-\boldsymbol{b}(t) \times \boldsymbol{G}_{\perp}(t)\right],
\end{gathered}
$$

where $\boldsymbol{G}_{\|}(t)$ and $\boldsymbol{G}_{\perp}(t)$ are respectively the density gradient components parallel and perpendicular to the IMF, $R_{\mathrm{L}}(t)$ is the particle's effective Larmor radius, $\boldsymbol{b}(t)$ is the unit vector pointing in the direction of the IMF, and $\alpha_{\|}$and $\alpha_{\perp}$ are the dimensionless mean free paths $\left(\lambda_{\|}(t)\right.$ and $\left.\lambda_{\perp}(t)\right)$ of GCR scattering by magnetic irregularities, defined as

$$
\begin{gathered}
\alpha_{\|}=\lambda_{\|}(t) / R_{\mathrm{L}}(t)=\left[3 \kappa_{\|}(t) / R_{\mathrm{L}}(t)\right] / c, \\
\alpha_{\perp}=\lambda_{\perp}(t) / R_{\mathrm{L}}(t)=\left[3 \kappa_{\perp}(t) / R_{\mathrm{L}}(t)\right] / c,
\end{gathered}
$$

where $\kappa_{\|}(t)$ and $\kappa_{\perp}(t)$ are respectively the parallel and perpendicular diffusion coefficients. We use for $R_{\mathrm{L}}(t)$ the Larmor radius of $50 \mathrm{GeV}$ protons in the IMF magnitude $B(t)$ in the $A C E$-Level2 


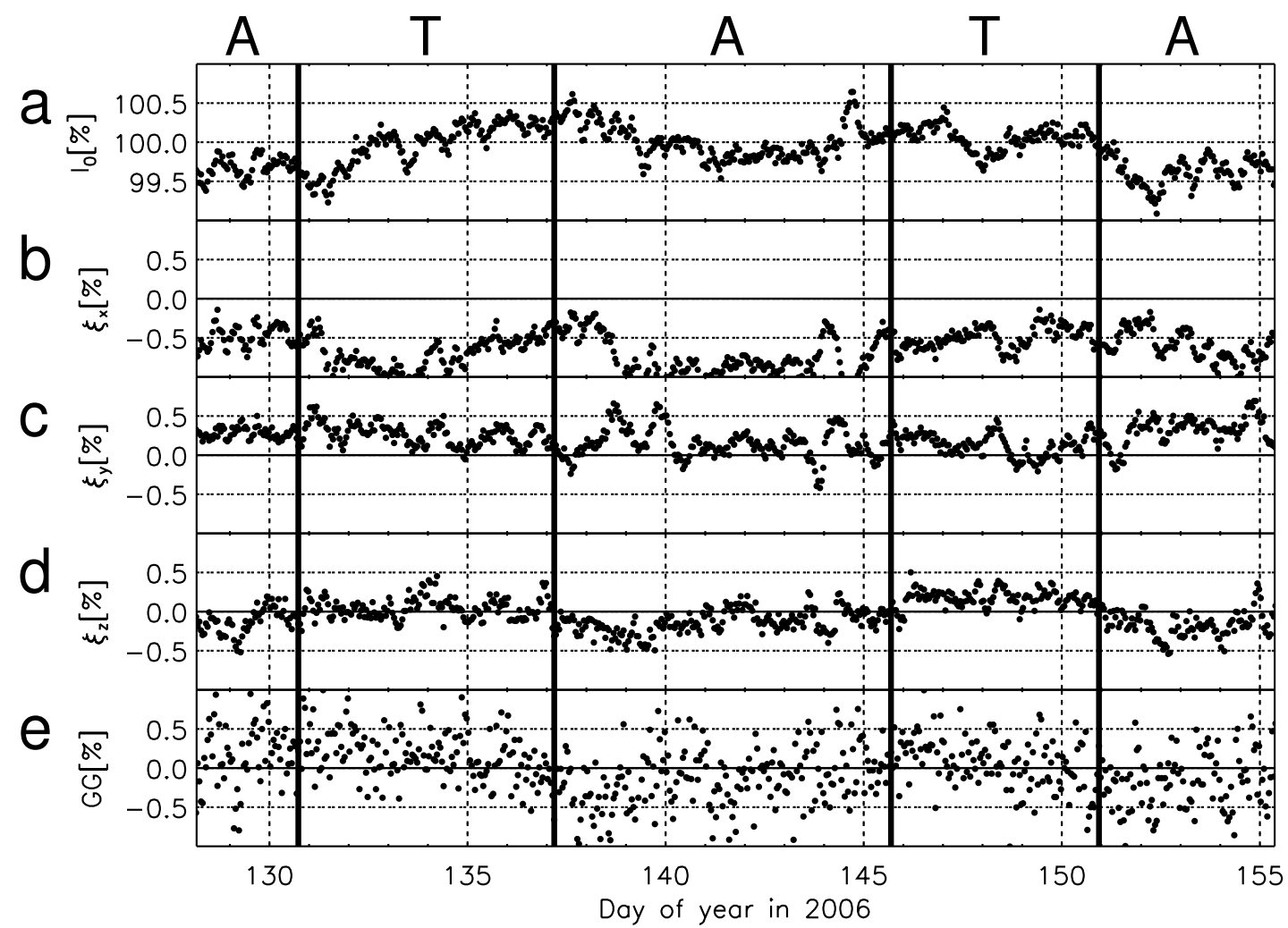

FIG. 2.-Best-fit anisotropy components in GSE coordinates for CR 2043 (2006 May 8 to June 4). The anisotropy derived in a local geographical coordinate system (GEO) is transformed to GSE coordinates and corrected for solar wind convection and the Compton-Getting effect (see text and Appendix A). From top to bottom, panels display (a) the best-fit density, $(b)-(d)$ the three components of the anisotropy, and (e) the hourly values of the GG component as a function of the day of year (DOY; see Appendix A for GG component). The time of the Earth's HCS crossing (as determined by the reversal of the IMF longitude) is indicated by the vertical solid line, while the polarity (T/A) of each IMF sector between the HCS crossings is indicated at the top of the figure.

data. The second term on the right-hand side of equation (4) expresses the drift anisotropy, while the first term represents the perpendicular diffusion. The parallel gradient $\boldsymbol{G}_{\|}(t)$ in equation (3) can be directly derived from $\boldsymbol{\xi}_{\|}(t)$ as

$$
\boldsymbol{G}_{\|}(t)=\left[\boldsymbol{\xi}_{\|}(t) / R_{\mathrm{L}}(t)\right] / \alpha_{\|}
$$

On the other hand, the form of equation (4) which applies only for the perpendicular component can be rewritten as

$$
\boldsymbol{\xi}_{\perp}(t)=R_{\mathrm{L}}(t)\left(\begin{array}{ccc}
\alpha_{\perp} & b_{z}(t) & -b_{y}(t) \\
-b_{z}(t) & \alpha_{\perp} & b_{x}(t) \\
b_{y}(t) & -b_{x}(t) & \alpha_{\perp}
\end{array}\right) \boldsymbol{G}_{\perp}(t) .
$$

By solving equation (8) with respect to $\boldsymbol{G}_{\perp}(t)$, we obtain

$$
\boldsymbol{G}_{\perp}(t)=\left(\begin{array}{ccc}
\alpha_{\perp} & b_{z}(t) & -b_{y}(t) \\
-b_{z}(t) & \alpha_{\perp} & b_{x}(t) \\
b_{y}(t) & -b_{x}(t) & \alpha_{\perp}
\end{array}\right)^{-1} \frac{\boldsymbol{\xi}_{\perp}(t)}{R_{\mathrm{L}}(t)} .
$$

This equation cannot be used for the case of $\alpha_{\perp}=0$, in which the inverse matrix in (9) becomes singular. In the case when crossfield diffusion is negligible relative to the drift streaming in equation (4), $\boldsymbol{G}_{\perp}(t)$ is given by

$$
\begin{aligned}
\boldsymbol{G}_{\perp}(t) & =\boldsymbol{b}(t) \times \boldsymbol{\xi}(t) / R_{\mathrm{L}}(t) \\
& =\left(\begin{array}{ccc}
0 & -b_{z}(t) & b_{y}(t) \\
b_{z}(t) & 0 & -b_{x}(t) \\
-b_{y}(t) & b_{x}(t) & 0
\end{array}\right) \frac{\boldsymbol{\xi}(t)}{R_{\mathrm{L}}(t)} .
\end{aligned}
$$

Ignoring the minor IMF component $\left(b_{z}\right)$ perpendicular to the ecliptic plane, we find in equation (10) that the NS anisotropy $\left[\xi_{z}(t)\right]$ in the GSE coordinate system reflects the ecliptic component of the gradient $\left[G_{\perp x}(t)\right.$ and $\left.G_{\perp y}(t)\right]$, while the anisotropy components in the ecliptic plane $\left[\xi_{x}(t)\right.$ and $\left.\xi_{y}(t)\right]$ are indicators of the NS gradient $\left[G_{\perp z}(t)\right]$. In the next section, we adopt two combinations of $\alpha_{\|}$and $\alpha_{\perp}$ to derive the gradient from the observed anisotropy. We first adopt an ad hoc choice of $\alpha_{\|}=7.2$ and $\alpha_{\perp}=0.36$, which will be used in the numerical calculation of a simple drift model. We then calculate $\boldsymbol{G}(t)$ with $\alpha_{\|}=0$ and $\alpha_{\perp}=0$ for the case of dominant drift streaming and compare the two.

\subsection{Density Gradient in a Sample Rotation}

Figure 3 shows the GCR density gradient derived from the anisotropy in Figure 2 by equations (7) and (9), together with the solar wind velocity and the IMF data in a sample rotation, CR 2043. From top to bottom, each panel displays $(a)$ the magnitude of the IMF, $(b)$ the solar wind velocity, $(c)$ the GSE longitude of the IMF, and $(d-f)$ the three GSE components of the derived gradient vector. The IMF sector boundaries and the sector polarities are indicated in the same manner as in Figure 2. A four-sector IMF structure is evident in this rotation. Enhancements of the IMF magnitude at the leading edges of the high-speed solar wind streams are also seen, indicating the CIR formed in the solar wind. Similar structures in the IMF and solar wind are observed over six solar rotations following CR 2043.

Figures $3 d-3 f$ display the GSE components of $\boldsymbol{G}_{\perp}(t)+\boldsymbol{G}_{\|}(t)$ calculated with $\alpha_{\|}=7.2$ and $\alpha_{\perp}=0.36$. These values were ad hoc selections and are primarily for illustration. Qualitative features would remain similar for a wide range of reasonable parameters. Each component shows a large fluctuation due to the 


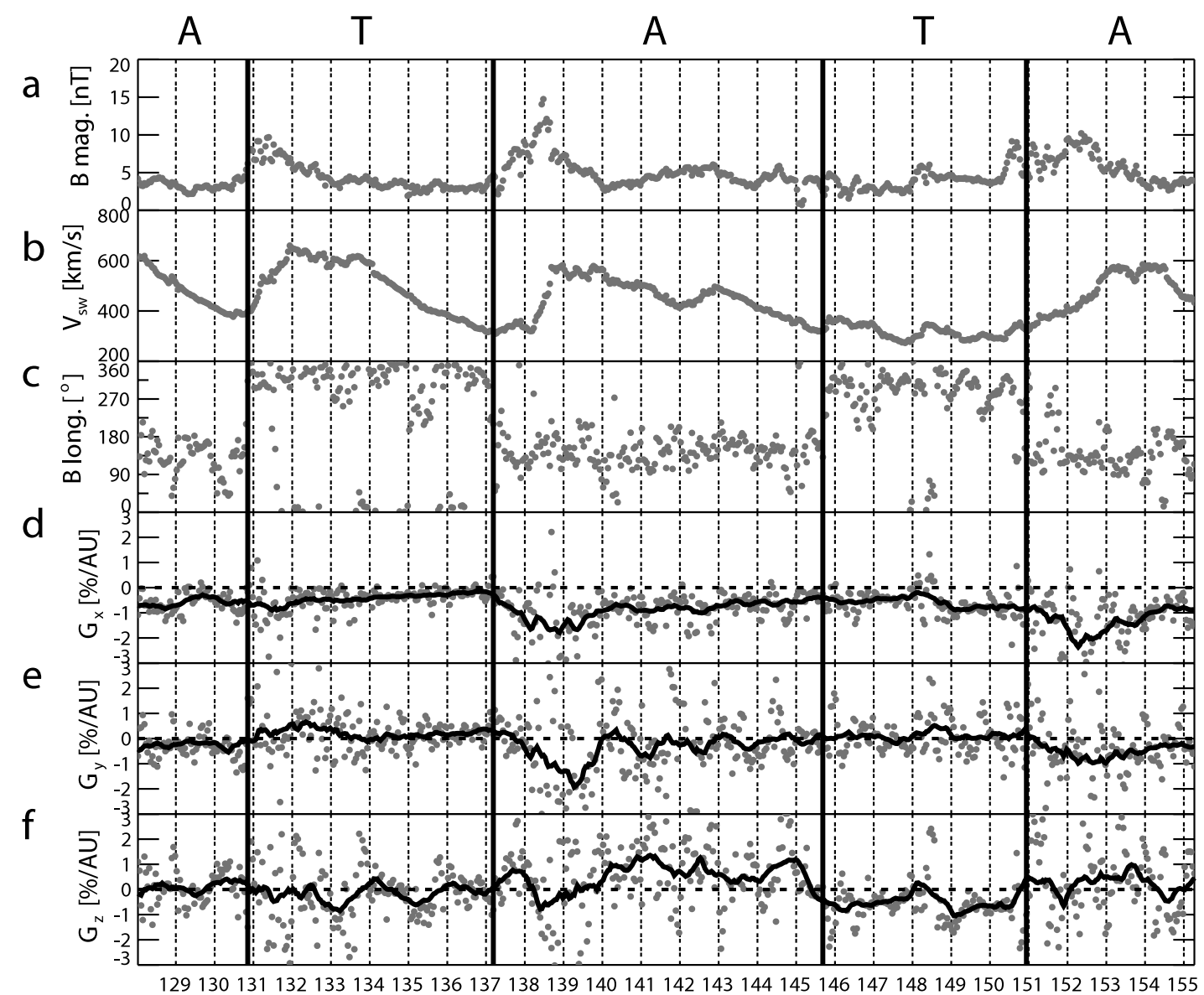

Day of year in 2006

FIG. 3.- Solar wind parameters and the GCR density gradient during CR 2043: (a) Magnitude of the IMF, $(b)$ solar wind speed, $(c)$ hourly mean GSE longitude of the IMF and three GSE components of the gradient, $(d) G_{x},(e) G_{y}$, and $(f) G_{z}$ derived with $\alpha_{\perp}=0.36$ and $\alpha_{\|}=7.2$ (see text). Panels $(d)-(f)$ display hourly values of the component of $\boldsymbol{G}_{\perp}(t)+\boldsymbol{G}_{\|}(t)$. Each black curve in $(d)-(f)$ displays the $23 \mathrm{hr}$ central moving average of the hourly gradient and shows the systematic variation of the gradient by filtering the large scattering of data points due to the fluctuation of the IMF orientation. The IMF sector boundaries and the sector polarities are indicated in the same manner as in Fig. 2.

fluctuation of the IMF orientation $\left(b_{x}, b_{y}, b_{z}\right)$ used in equations (2) and (9). To show the systematic variation of each component, we filter this fluctuation with a central 23 hour moving average of hourly data, plotted as the black curve. This systematic variation is dominated by the contributions from $\boldsymbol{G}_{\perp}(t)$, the contribution from $\boldsymbol{G}_{\|}(t)$ being less than $20 \%$ on average. The following correlations with IMF sector structure are apparent. First, $G_{z}(t)$ in Figure $3 f$ tends to be negative (positive) in T (A) sectors. This relationship between the sign of $G_{z}(t)$ and the IMF sector polarity is schematically shown in Figure 4. During $A<0$ epochs, the IMF points away from the Sun below the HCS (i.e., on the southern side of the HCS). The positive (negative) $G_{z}(t)$ in A (T) sectors indicates that the gradient is northward below the HCS (Fig. 4a), while it is southward above the HCS (Fig. 4b). This implies that the GCR density distribution has a local maximum at the HCS. This is in a qualitative agreement with drift model predictions for $A<0$ epochs. By analyzing the data observed with ground-based detectors such as NMs and muon detectors, the bidirectional NS density gradient has previously only been examined for drift effects on a yearly basis. The GMDN successfully observed it as a dynamic variation of the density gradient within a solar rotation.

We next note, in Figures $3 d$ and $3 e$, that the ecliptic component $G_{x}(t)$ remains negative most of the time and shows enhancements following HCS crossings on DOYs 137 and 150. Such enhancements are also seen in $G_{y}(t)$. The negative $G_{x}(t)$ implies that the (a)

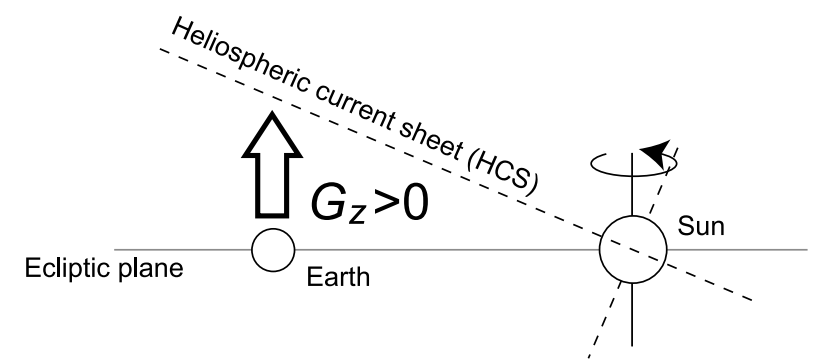

(b)

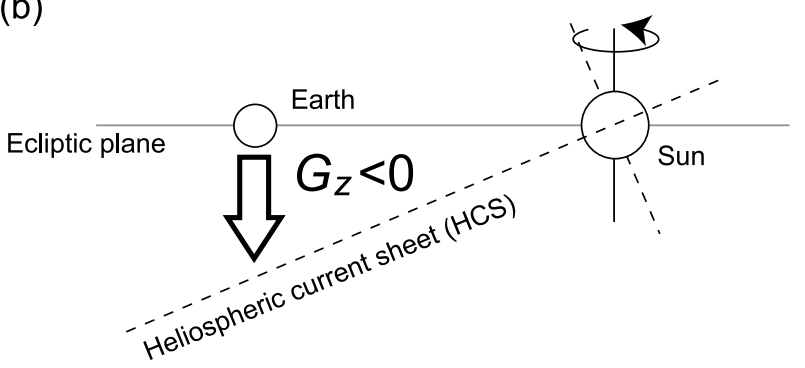

FIG. 4.- Schematic view in the meridian plane of $G_{z}$ changing sign according to the IMF sector polarity. Panels $a$ and $b$ represent situations when the Earth is on the southern side of the HCS (A sector) and when it is on the northern side ( T sector), respectively. 
(a)

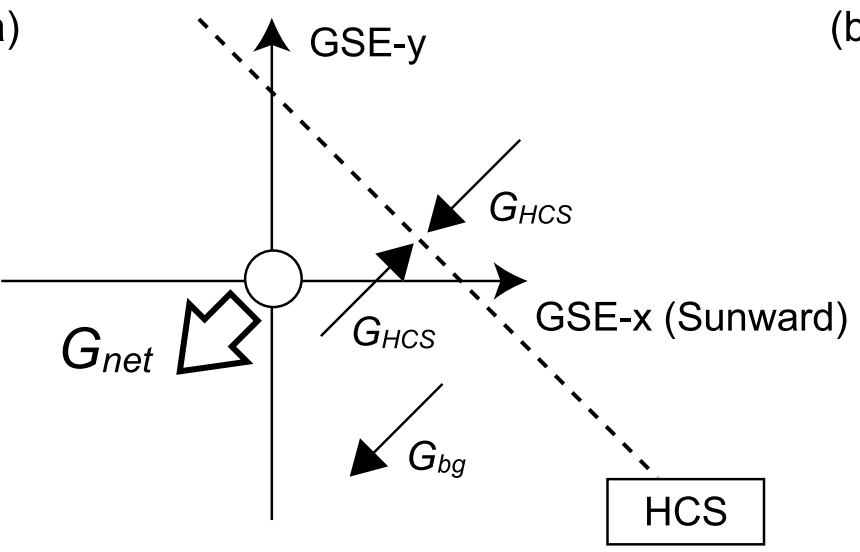

(b)

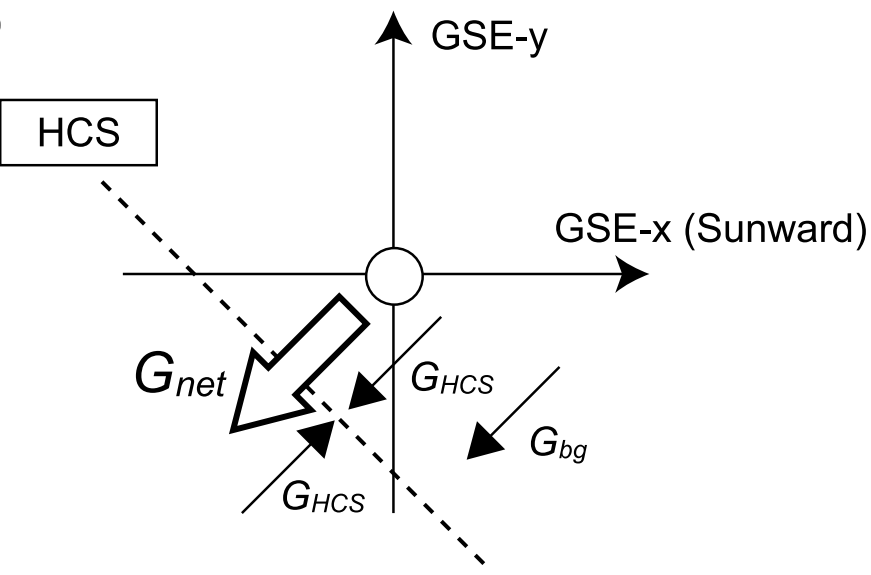

FIG. 5.- Schematic view of the ecliptic component of $\boldsymbol{G}$ varying in association with the Earth's HCS crossing. Panels $a$ and $b$ represent situations on the ecliptic plane before and after the HCS crossing, respectively. The figure displays only the gradient components that are perpendicular to the line of intersection of the HCS with the ecliptic plane.

ambient radial density gradient is positive and the density increases away from the Sun in response to the outward convection by the solar wind. As shown in Figure 5, the enhancement of the ecliptic component of $\boldsymbol{G}(t)$ can be interpreted in terms of the bidirectional density gradient toward the $\mathrm{HCS}\left(G_{\mathrm{HCS}}\right)$ in the ecliptic plane, together with the contribution from the ambient radial gradient away from the $\operatorname{Sun}\left(G_{\mathrm{bg}}\right)$. In this situation, $G_{\mathrm{HCS}}$ observed at the Earth is opposite to $G_{\mathrm{bg}}$ before the HCS crossing (Fig. $5 a$ ), while $G_{\mathrm{HCS}}$ and $G_{\mathrm{bg}}$ point in the same direction after the crossing (Fig. $5 b)$. The resultant density gradient $\left(G_{\text {net }}\right)$ observed at the Earth thus increases after the HCS crossing. This is observed as enhancements of $G_{x}(t)$ and $G_{y}(t)$ in association with HCS crossings. These enhancements therefore also imply a local maximum of the GCR density at the HCS. We finally demonstrate that the systematic variations discussed above are mainly due to drift streaming. Gray curves in Figure 6 show the three components of $\boldsymbol{G}(t)$ calculated by (10) for $\alpha_{\perp}=0$ and $\alpha \|=0$. Note that contributions from the parallel and cross-field diffusions are disregarded in this case. Nevertheless, the variations of $G_{x}(t)$ and $G_{y}(t)$ are not significantly different from those for $\alpha_{\perp}=0.36$ and $\alpha_{\|}=7.2$, shown by black curves. It follows that the observed systematic variations in $\boldsymbol{G}(t)$ are predominantly caused by drift streaming.

\subsection{Comparison with a Simple Drift model}

In this subsection, we compare the observed gradients with the numerical prediction of a simple drift model. The GCR density distribution is calculated by numerically solving Parker's diffusive transport equation (Parker 1965) in a three-dimensional model heliosphere. The model incorporates all the important physical processes: anisotropic diffusion, convection, particle drifts and adiabatic cooling in the expanding solar wind, as well as acceleration at shocks or in compression regions. The code assumes steady-state conditions in the frame corotating with the Sun. The code is able to handle complex shapes of the current sheet and nonuniform solar wind speed leading to the formation of CIRs. In this work, for brevity, we take a simple constant $400 \mathrm{~km} \mathrm{~s}^{-1}$ solar wind, so the effects of CIRs are not considered. We adopt a slightly warped current sheet, which leads to an asymmetric two-sector field. The tilt of the sheet is $\sim 15^{\circ}$, consistent with the average tilt angle of $12.6^{\circ}$ computed from the photospheric magnetic field by the radial field model for CR $2043 .{ }^{12}$ The areas above and below the current sheet are equal, and the radial magnetic

12 See http://wso.stanford.edu.

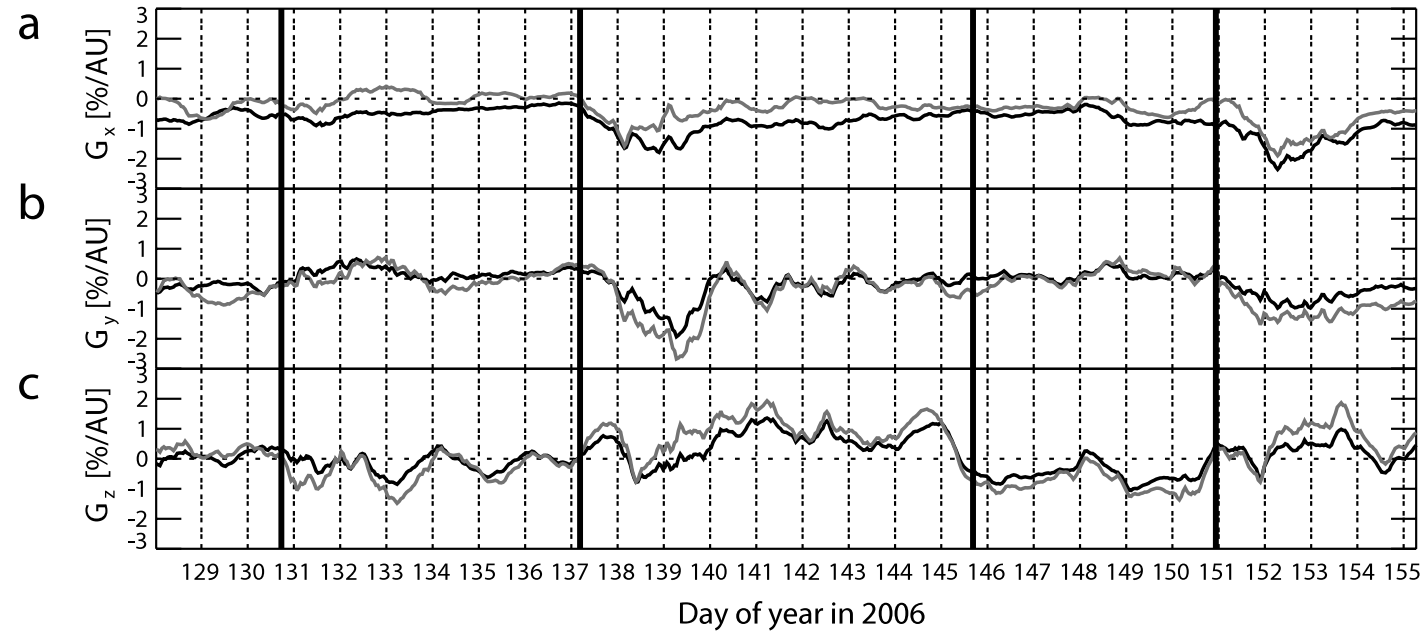

FIG. 6.- Variations of the three GSE components of $\boldsymbol{G}$ for different $\alpha_{\perp}$ and $\alpha_{\|}:(a) G_{x},(b) G_{y}$, and $(c) G_{z}$. In each panel, the black line displays $\boldsymbol{G}(t)$ for $\alpha_{\perp}=0.36$ and $\alpha_{\|}=7.2$, while the gray line shows $\boldsymbol{G}(t)$ for $\alpha_{\perp}=0$ and $\alpha_{\|}=0$ for the pure drift-streaming case. The timing of each HCS crossing is indicated by the vertical line. 


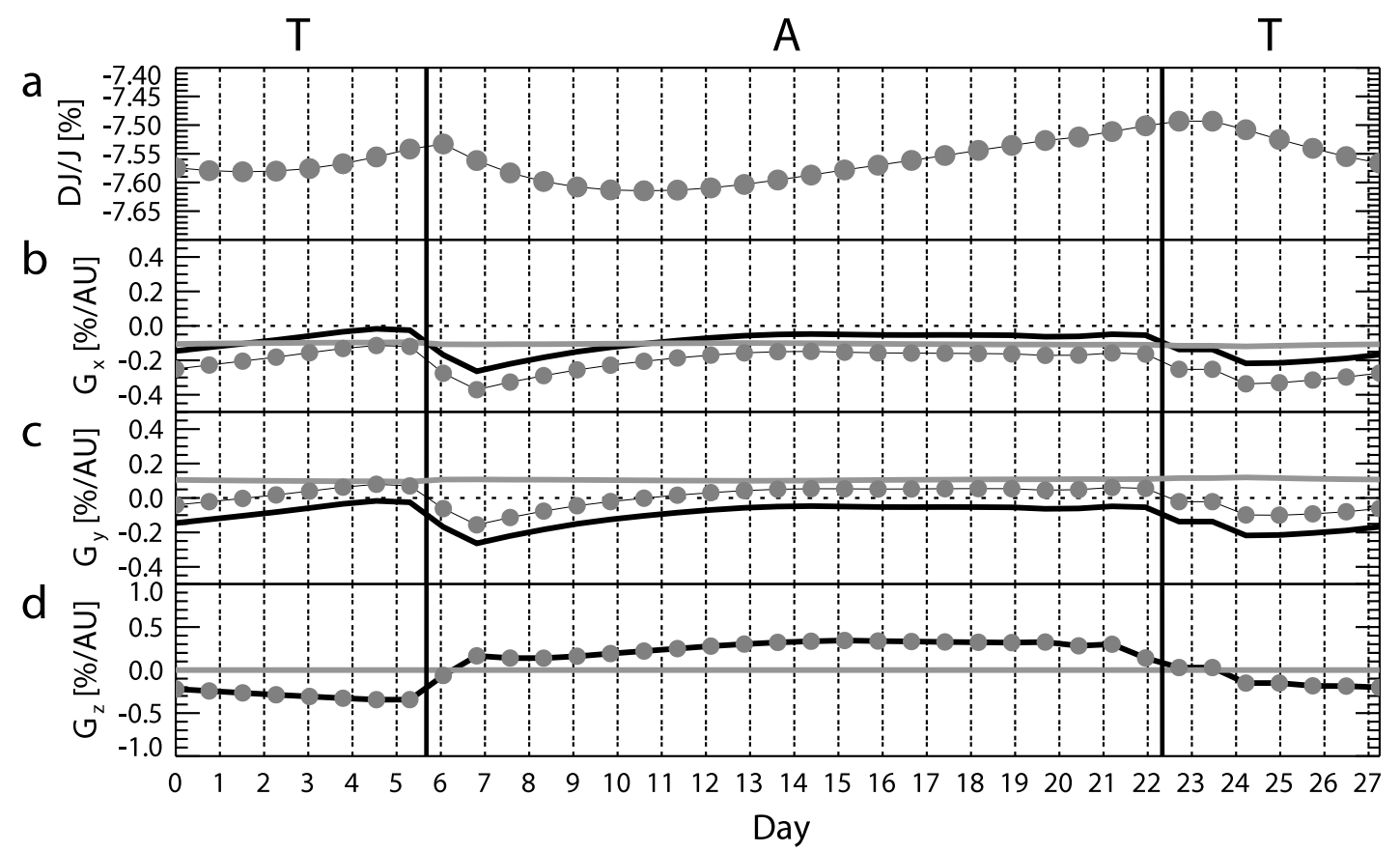

Fig. 7.-27 day variation in the gradient, $\boldsymbol{G}$, of $50 \mathrm{GeV}$ GCRs calculated numerically from a simple three-dimensional drift model for $A<0$. The model assumes $\alpha_{\perp}=0.36$ and $\alpha_{\|}=7.2$ and two IMF sectors separated by a wavy HCS with a tilt angle of $15^{\circ}$. No CIRs are included. From top to bottom, panels display $(a)$ the GCR density normalized to the interstellar value and $(b)-(d)$ the three GSE components of $\boldsymbol{G}$. Gray filled circles connected by a thin line in each panel display the hourly values. In panels $b-$ $d$, the black trace displays the component of $\boldsymbol{G}_{\perp}(t)$, while the gray trace shows the contribution of $\boldsymbol{G}_{\|}(t)$. The sector boundary is indicated by the vertical line, while the polarity ( $\mathrm{T} / \mathrm{A})$ of each IMF sector is indicated at the top of the figure.

field component is set uniformly at $3.5 \mathrm{nT}$ at $1 \mathrm{AU}$, while the total field in the helioequator at $1 \mathrm{AU}$ (near the Earth) is $5 \mathrm{nT}$.

The code has been updated to calculate GCR gradients and anisotropies (see Kóta \& Jokipii 2001). At high energies (tens of $\mathrm{GeVs}$ ) the relative energy change of GCRs in the heliosphere is small; hence the spectral exponent remains equal to its interstellar value, and can be taken as a prescribed constant. The important parameters to determine the anisotropies are the ratio of the three components of the diffusion tensor,

$$
\kappa_{\|}: \kappa_{A}: \kappa_{\perp}=\alpha_{\|}: 1: \alpha_{\perp}
$$

Assuming that all these elements of the diffusion tensor scale inversely with the magnetic field strength, $B$, and are proportional to rigidity, these ratios remain constant. In this case, gradients will scale inversely proportional to the particle rigidity while anisotropies remain constant, as long as the diffusive description is appropriate. The diffusive approach becomes inaccurate and should break down at some limiting rigidity; the transition between diffusive and nondiffusive behaviors is not yet well understood (Kóta 1999).

In the model simulation used in this work, we adopt $\kappa_{\|}=$ $2.4 R_{\mathrm{L}} c$ and $\kappa_{\perp}=0.12 R_{\mathrm{L}} c$, corresponding to $\alpha_{\|}=7.2$ and $\alpha_{\perp}=$ 0.36 . Comparison with more complex models including four-sector fields and CIRs are in progress and will be addressed in future work.

Figure 7 shows three components of the calculated density gradient of $50 \mathrm{GeV} \mathrm{GCRs} \mathrm{as} \mathrm{measured} \mathrm{at} \mathrm{the} \mathrm{Earth} \mathrm{over} \mathrm{one} \mathrm{so-}$ lar rotation period during an $A<0$ epoch. In each panel of Figures $7 b-7 d$, gray filled circles connected by thin lines display the GSE component of the total gradient $\boldsymbol{G}_{\perp}(t)+\boldsymbol{G}_{\|}(t)$, while contributions from $\boldsymbol{G}_{\perp}(t)$ and $\boldsymbol{G}_{\|}(t)$ are shown by black and gray curves, respectively. Although the calculated contribution from $\boldsymbol{G}_{\|}(t)$ is somewhat larger, it is apparent that the temporal varia- tion of the total gradient is mainly due to $\boldsymbol{G}_{\perp}(t)$, as in Figure 3 . The overall magnitude of the calculated gradient is also in reasonable agreement with the observations, except in the periods of observed strong enhancements following HCS crossings. The observed systematic variations in the three components of the gradient are well reproduced by the simple drift model. First, $G_{z}(t)$ is negative (positive) in T (A) sectors (see Fig. 7d). Second, the ecliptic component $G_{x}(t)$ remains negative during the entire rotation period and shows enhancements after HCS crossings. Similar enhancements are also seen in $G_{y}(t)$ (see Figs. $7 b-7 c$ ). These similarities between the observations and the model support drifts being responsible for the observed modulation of GCRs in the sample rotation period. In the next section we discuss the similarity between the observed and predicted gradients in more detail.

Figure 3 shows that $G_{z}(t)$ is negative (positive) in T (A) sectors. On the other hand, $G_{x}(t)$ and $G_{y}(t)$ show these features in only two sectors starting from DOYs 137 and 151, but not in two other sectors starting from DOYs 130 and 145 . There is no enhancement in the solar wind speed and IMF after DOY 145, while there are clear enhancements after DOY 130 . We currently have no clear interpretation of this. It also appears that $G_{x}(t)$ and $G_{y}(t)$ only show the above-mentioned feature after HCS crossings from $\mathrm{T}$ sectors to A sectors. If this is the case, it may be due to some asymmetry between the northern ( $\mathrm{T}$ ) and southern (A) hemispheres that is not taken into account in the present simple drift model.

\section{SUMMARY AND DISCUSSION}

We derived the GCR density gradient in three dimensions from the anisotropy observed with the GMDN, which has been operating since March 2006. In particular, we have developed a new analysis method for deducing the anisotropy free from atmospheric temperature effects on the muon intensity recorded by 
ground-based detectors. The new method, together with the global sky coverage available in the GMDN, allows us to accurately derive the anisotropy in three dimensions, including the northsouth anisotropy, which has previously been difficult to analyze for timescales less than a day. We analyze the anisotropy observed in 13 solar rotations between 2006 March 14 and 2007 March 4. During this period around solar activity minimum, clear signatures of recurrent CIRs were seen with enhancement of the IMF magnitude at the leading edge of high-speed solar wind streams from coronal holes on the solar surface.

Solving the diffusion flux equation inversely for the spatial gradient of the GCR density, we deduced the gradient in three dimensions from the observed anisotropy corrected for both the solar wind convection and the Compton-Getting effect arising from the Earth's orbital motion. By analyzing the gradient derived in a typical sample rotation (CR 2043), we find that the GSE $z$ component $G_{z}(t)$ of the gradient is negative (positive) in T (A) sectors. On the other hand, the GSE $x$ component $G_{x}(t)$ remains negative most of the time, indicating that the ambient radial gradient is positive with the GCR density increasing away from the Sun in response to the outward convection by the solar wind. Significant enhancements are also seen in both $G_{x}(t)$ and $G_{y}(t)$ following HCS crossings. These features are seen both in the sample rotation and in the average of the 13 rotations observed. Statistical analyses of multiple rotations will be presented in a separate paper. We demonstrate that these features can be interpreted in terms of the local maximum of GCR density at the HCS which is consistent with the prediction of the drift model for $A<0$ epochs. It is noted from Figure 6 that similar features are also seen in the gradient derived when neglecting the contribution from the parallel and perpendicular diffusion. This confirms that the observed anisotropy is dominated by drift streaming at these high energies.

We also compare these features with the numerical solution of the GCR transport equation in the three-dimensional heliosphere with a simple two-sector IMF structure. There is an overall resemblance between the observed gradient and the prediction by this simple model, ignoring the CIR signatures such as strong enhancements of both the solar wind velocity and the IMF magnitude. This comparison with the model is preliminary. The model does not take into account effects of CIRs. In particular, the enhanced IMF magnitude in the CIR leads to a reduced Larmor radius $R_{\mathrm{L}}(t)$ and an enhanced gradient by a factor of $1 / R_{\mathrm{L}}(t)$, as seen in equations (9) and (10). By comparing gradients derived employing an IMF with and without this magnitude enhancement, we confirmed that this effect contributed about $50 \%$ of the enhanced gradient. The remaining half of the enhancement still exists, even without the IMF enhancement, with temporal variations similar to those above. The model we used in this work also assumes a two-sector IMF structure, while the observed sample rotation clearly shows the four-sector structure. The average features of the gradient mentioned above are observed in only two sectors, but are not observed in the other sectors. Further analyses based on a more realistic model are needed to clarify this.

The discussions above suggest that the GCR modulation by CIRs does not solely organize the observed gradient, but the drift effect in the large-scale magnetic field plays a more important role. This seems to contradict the conclusion, derived from satellite measurements of sub-GeV particles, that the GCR modulation is not organized by the HCS (Richardson 2004). A possible explanation for this discrepancy is the difference in particle energy. Sub-GeV GCRs have smaller Larmor radii and are more sensi- tive to small-scale structures in the solar wind. Conversely, the primary GCRs to which GMDN network responds are much more energetic $(\sim 50-150 \mathrm{GeV})$ and less sensitive to small-scale structures, selectively responding to the larger scales over which the global drift mechanism operates. It is also reported in a recent paper by Minnie at al. (2007) that drifts are suppressed by magnetic turbulence, but the suppression sets in at lower turbulence amplitudes for low-energy cosmic rays than for high-energy cosmic rays. This may give a possible explanation for why drift effects might be dominant at muon detector energies, but negligible at sub-GeV energies. The difference in the analysis method may also be responsible for the discrepancy. Analyses reported by Richardson (2004) were based on a one-dimensional spatial distribution of the GCR density along the viewing path of a single detector moving through the solar wind. A single detector measurement of the density cannot, however, observe the spatial distribution separately from the temporal variation of the density at a particular location in space. The spatial distribution sampled by a single detector also does not reflect the global distribution surrounding the detector, above and below the detector's viewing path. It is also affected by local irregularities in the solar wind. This can be serious, particularly when analyzing satellite measurements of low-energy (sub-GeV) GCRs. Our analysis, on the other hand, uses the GCR spatial gradient in three dimensions, which provides us with additional information on the distribution around the detectors' viewing paths. It is not always easy to deduce the large-scale distribution of GCRs solely from the temporal variation of the density, particularly when the amplitude of the variation is small. The modulation of the GCR density in the CIR observed during the solar minimum period is generally small, when compared with other violent events such as the Forbush decrease caused by the Earth's encounter with a coronal mass ejection (CME) accompanied by a strong shock. This is seen in the best-fit density in Figure $2 a$. The modulation of the density in this figure is less than $1 \%$, with no clear correlation with HCS crossings and/or the CIR structures in Figures $3 a-3 c$.

In conclusion, we demonstrate that both the observation of high-energy GCRs with the GMDN and employing a new analysis method provide us with new information about the density gradient in three dimensions. In particular, we find that the gradient observed in a sample solar rotation period shows systematic variations correlated with the Earth's HCS crossings. These features are in good agreement with the prediction of a simple drift model for $A<0$ epochs. However, they are only seen in two of the four sectors observed in the sample rotation period. Clarifying the physical mechanism responsible for this requires further analyses, including statistical analysis of multiple rotations and detailed comparisons using more realistic model calculations. Analysis of the gradient observed by the GMDN in association with CIR structures in $A>0$ epochs will give us a unique opportunity to test the drift model.

This work is supported in part by US NSF grant ATM-0527878 and NASA grant NNX 07-AH73G, and in part by Grants-in-Aid for Scientific Research from the Ministry of Education, Culture, Sports, Science and Technology in Japan and by the joint research program of the Solar-Terrestrial Environment Laboratory, Nagoya University. The observations with the Kuwait University muon detector are supported by the Kuwait University grant SP03/03. We thank N. F. Ness for providing $A C E$ magnetic field data via the $A C E$ Science Center. 


\section{APPENDIX A}

\section{DATA ANALYSIS METHOD AND PERFORMANCE}

Muon detectors measure high-energy Galactic cosmic rays (GCRs) by detecting secondary muons produced from the hadronic interactions of primary GCRs (mostly protons) with atmospheric nuclei. Since relativistic muons have relatively long lifetimes (the proper half-life is $2.2 \mu \mathrm{s}$ ) and can reach the ground preserving the incident direction of the initiating primary particles, we can measure the GCR intensity in various directions with a multidirectional detector at a single location. This is different from neutron monitor (NM) measurements, where directional information is only determined by the geomagnetic field at the time of the observation and the rigidity of the initiating primary.

The observed intensity of muons at ground level is subject to atmospheric temperature effects. Atmospheric expansion with increasing temperature causes an increase of the mean altitude of muon production from the decay of parent pions. The increased flight time results in the decay of more muons into electrons and neutrinos before they reach the detector. This effect is known as the "negative temperature effect" and causes a decrease (increase) of the muon count rate with increasing (decreasing) temperature. Kuwabara et al. (2004) eliminated the temperature effect by normalizing the $24 \mathrm{hr}$ trailing moving average (TMA) of the muon intensity recorded in each directional channel to that in the vertical channel of one of the muon detectors in the network. This method works well when we analyze the dynamically changing anisotropy within a day in association with the coronal mass ejection (CME) arrival at Earth. However, as shown below, it does not give correct results in the derivation of the north-south (NS) anisotropy and its relationship to the interplanetary magnetic field (IMF) sector structure.

Nagashima et al. (1972) proposed an observational method to measure the NS anisotropy by taking the difference between muon intensities in the north- and south-viewing channels in a multidirectional muon detector at Nagoya in Japan. By taking the difference, the atmospheric temperature effects common to all directional channels are removed (Sagisaka 1986). The difference is called the "GG component" (see eq. [A10]). The NS anisotropy represented by the average GG component has been regarded as arising from the drift streaming expressed by $\boldsymbol{B} \times \boldsymbol{G}_{r}$, where $\boldsymbol{G}_{r}$ is the radial density gradient. This streaming reverses in association with the reversal of $\boldsymbol{B}$ when the Earth crosses the HCS, as $\boldsymbol{G}_{r}$ remains pointing away from the Sun on average (Swinson 1969). The GG component is regarded as a good indicator of the IMF sector polarity. It has been reported, from a comparison between in situ measurements of the IMF polarity and the sign of the GG component, that the IMF polarity can be inferred from the GG component with an overall success rate exceeding $70 \%$ on a daily basis (Mori \& Nagashima 1979; Laurenza \& Storini 2003). In many cases, however, the GG component can only be used after averaging over a one day period. This is due to the diurnal anisotropy also contributing to the GG component. The diurnal anisotropy, when observed by a detector on the Earth, produces a diurnal variation in which the muon rate in each directional channel varies as a sinusoidal function of local time. Since the amplitude and local time of maximum of this diurnal variation is generally different in different directional channels, the variation remains present even in the GG component. Only after averaging over one day does the GG component become free of the contribution from the stationary diurnal anisotropy. Therefore, the GG component from a single muon detector at Nagoya cannot be used for monitoring the NS anisotropy for periods shorter than one day.

In the following subsections, we first show how the temperature effect appears in the anisotropy derived from the GMDN observations. In particular, we demonstrate that the conventional method employed for eliminating the temperature effect diminishes the north-south anisotropy as well. We then develop a new analysis method and apply it to the GMDN data.

\section{A1. DERIVATION OF THE COSMIC RAY ANISOTROPY IN THREE DIMENSIONS}

We derive the anisotropy by fitting the function $I_{i, j}^{\text {fit }}(t)$ given by

$$
I_{i, j}^{\mathrm{fit}}(t)=I_{i, j}^{0}(t)+\xi_{x}^{\mathrm{GEO}}(t)\left(c_{1 i, j}^{1} \cos \omega t_{i}-s_{1 i, j}^{1} \sin \omega t_{i}\right)+\xi_{y}^{\mathrm{GEO}}(t)\left(s_{1 i, j}^{1} \cos \omega t_{i}+c_{1 i, j}^{1} \sin \omega t_{i}\right)+\xi_{z}^{\mathrm{GEO}}(t) c_{1 i, j}^{0}
$$

to the observed hourly count rate $I_{i, j}^{\mathrm{obs}}(t)$ of muons at universal time $t$ in the $j$ th directional channel of the $i$ th muon detector in the $\operatorname{GMDN}$ (Kuwabara et al. 2004). In this equation, $\xi_{x}^{\mathrm{GEO}}(t), \xi_{y}^{\mathrm{GEO}}(t)$, and $\xi_{z}^{\mathrm{GEO}}(t)$ are the best-fit parameters denoting three components of the anisotropy; $c_{1 i, j}^{1}, s_{1 i, j}^{1}$, and $c_{1 i, j}^{0}$ are the coupling coefficients calculated by assuming a rigidity-independent anisotropy; $t_{i}$ is the local time at the location of the $i$ th detector, and $\omega=\pi / 12$. The anisotropy components in equation (A1) are defined in a local geographical coordinate system (GEO), in which the $z$-axis is directed toward geographic north, the $x$-axis is in the equatorial plane and directed to the zenith of a point on the Earth's equator at 00:00 local time, and the $y$-axis completes the right-handed coordinate set. Note that the anisotropy vector points toward a direction from which the highest GCR flux is measured; i.e., the anisotropy vector is oppositely directed to the GCR streaming vector. Among the three components of the anisotropy, $\xi_{z}^{\mathrm{GEO}}(t)$ represents the north-south (NS) anisotropy. We assume $I_{i, j}^{0}(t)$ as

$$
I_{i, j}^{0}(t)=I_{0}^{\prime}(t)+I_{0}^{\prime \prime}(t) P_{m i, j} / P_{m 1,1}
$$

where $I_{0}^{\prime}(t)$ and $I_{0}^{\prime \prime}(t)$ are best-fit parameters and $P_{m i, j}$ and $P_{m 1,1}$ are the median primary rigidities $\left(P_{m}\right.$ in Table 1$)$ of the $j$ th directional channel of the $i$ th muon detector and Nagoya vertical channel $(i=1, j=1)$, respectively. The first term on the right-hand side represents the energy-independent portion of the density, while the second term represents the rigidity dependence. We define the best-fit density $I_{0}(t)$ as

$$
I_{0}(t)=I_{0}^{\prime}(t)+I_{0}^{\prime \prime}(t)
$$




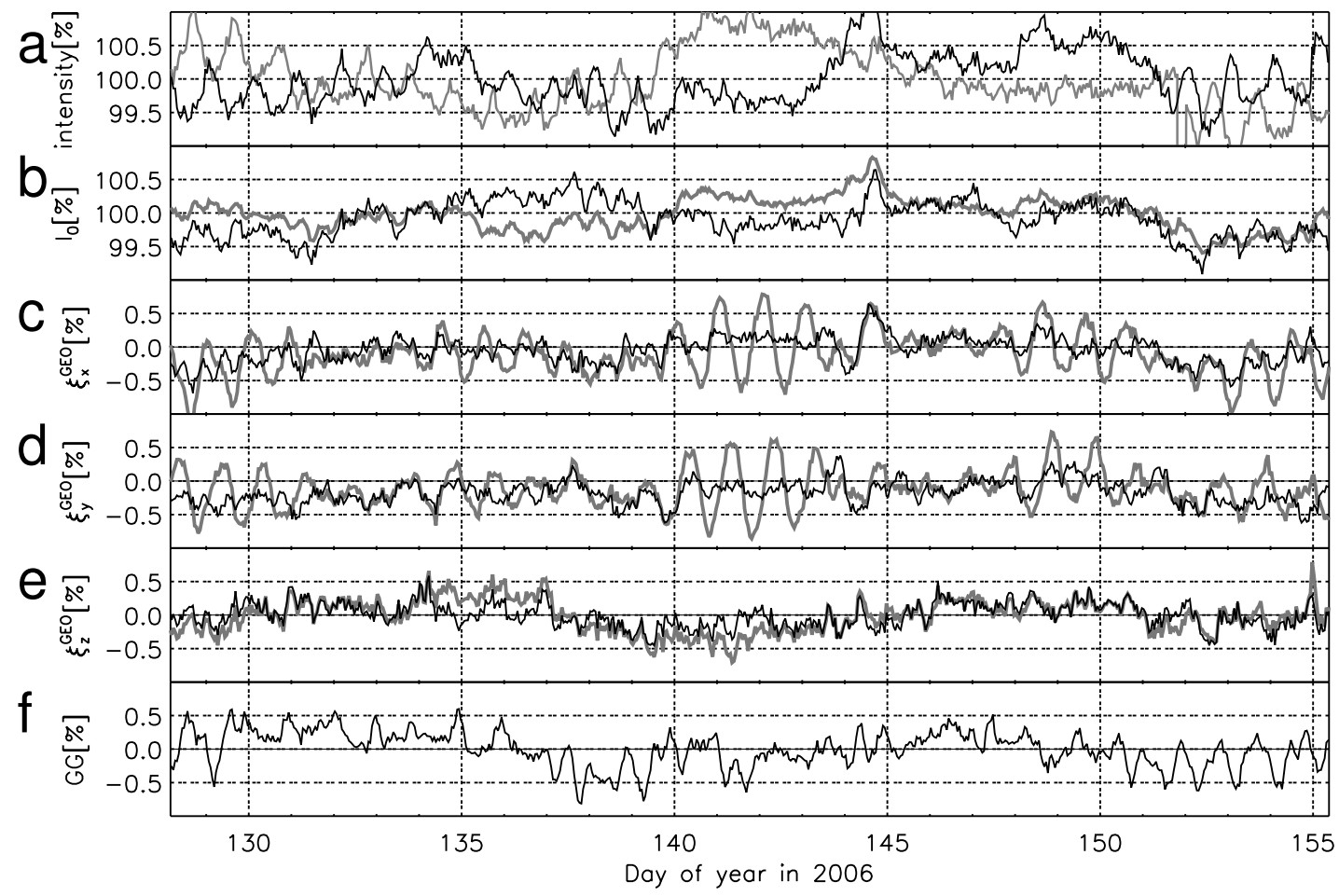

FIG. 8. - Best-fit parameters in eq. (A1) derived from best fits to the hourly GMDN data in a sample solar rotation period, CR 2043. From top to bottom, (a) the pressurecorrected vertical muon rates, $(b)$ the best-fit density, $(c)-(e)$ the three components of anisotropy in the GEO, and $(f)$ the Nagoya GG component are shown plotted against time (DOY). The black and thin black curves in $(a)$ show respectively the vertical muon rates at Nagoya and São Martinho. The gray curves in $(b)-(e)$ display parameters obtained from best fitting to the original muon rate, while the black curves in $(c)-(e)$ show parameters by the new analysis method. Note that the best-fit density in $(b)$ cannot be directly derived from the new analysis method. The density by the new method displayed by a gray curve in $(b)$ is derived by using the vertical muon rate at Nagoya, corrected for the atmospheric temperature effects (see text). The bottom panel $(f)$ shows the $5 \mathrm{hr}$ central moving average of the hourly value of the GG component.

which is equal to $I_{1,1}^{0}(t)$ for the vertical channel of Nagoya with $P_{m}=59 \mathrm{GV}$. We determine the best-fit set of parameters $\left[I_{0}(t), \xi_{x}^{\mathrm{GEO}}(t)\right.$, $\left.\xi_{y}^{\mathrm{GEO}}(t), \xi_{z}^{\mathrm{GEO}}(t)\right]$ that minimize the residual $S$, defined as

$$
S=\sum_{i, j}\left[I_{i, j}^{\mathrm{obs}}(t)-I_{i, j}^{\mathrm{fit}}(t)\right]^{2} / \sigma_{c i, j}^{2}
$$

where $\sigma_{c i, j}$ is the count rate error in the $j$ th directional channel of the $i$ th muon detector $\left(\sigma_{c}\right.$ in Table 1).

We first fit equation (A1) to the observed percentage deviation $r_{i, j}^{\text {obs }}(t)$ of the pressure-corrected hourly counting rate from the average over each solar rotation. Gray curves in Figures $8 b-8 e$ display the best-fit density and three GEO components of the anisotropy, while Figure $8 a$ shows the observed muon count rates $\left[r_{i, 1}^{\text {obs }}(t)\right]$ in vertical channels at Nagoya (thicker black curve) and São Martinho (thin black curve), each as a function of the day of year (DOY) in a sample rotation period (CR 2043) in 2006. In Figure $8 a$ we plot muon rates in two vertical channels to demonstrate the atmospheric temperature effect, but the parameters in Figures $8 b-8 e$ are derived from best-fitting to data from all directional channels in the GMDN.

As illustrated by the gray curves in Figures $8 b-8 e$, best-fitting to the original percent deviation $r_{i, j}^{\mathrm{obs}}(t)$ results in large diurnal variations in the anisotropy, particularly when the difference between the vertical muon rates at Nagoya and São Martinho in Figure $8 a$ becomes significant. This is due to the atmospheric temperature effect described in Appendix B, simply because best-fitting the model intensity in equation (A1) leads to an anisotropy from the direction with the higher count rate. For instance, as seen in Figure $8 a$ in DOYs 140-144, the vertical muon rate is higher at São Martinho than at Nagoya, while the best-fit anisotropy component $\xi_{x}^{\mathrm{GEO}}(t)$ in Figure $8 c$ shows a maximum around 03:00 UT when the viewing directions of the São Martinho muon detector point toward the GEO $x$ direction. On the other hand, when the vertical muon rate is higher at Nagoya than at São Martinho (e.g., DOY 148-151), the best-fit $\xi_{x}^{\mathrm{GEO}}(t)$ shows a maximum around 14:00 UT, corresponding to the time when the viewing directions at Nagoya point toward GEO- $x$.

To avoid this spurious diurnal variation in the derived anisotropy, Kuwabara et al. (2004) calculated $I_{i, j}^{\text {obs }}(t)$ for the best-fit by normalizing the $24 \mathrm{hr}$ trailing moving average (TMA) of $r_{i, j}^{\mathrm{obs}}(t)$ to that of the vertical channel in the Nagoya muon detector as

$$
I_{i, j}^{\mathrm{obs}}(t)=r_{i, j}^{\mathrm{obs}}(t) \bar{r}_{1,1}(t) / \bar{r}_{i, j}(t)
$$

where $\bar{r}_{i, j}(t)$ is the $24 \mathrm{hr}$ TMA of $r_{i, j}(t)$, defined as

$$
\bar{r}_{i, j}(t)=\sum_{t-23}^{t} r_{i, j}^{\mathrm{obs}}(t) / 24,
$$


and $\bar{r}_{1,1}(t)$ is the TMA for the vertical channel at Nagoya. This normalization efficiently removes the spurious diurnal variation of the bestfit anisotropy and works well for deriving the temporal variation over a period shorter than $24 \mathrm{hr}$. It has been used for analyzing the geometry of a CME (Kuwabara et al. 2004). The normalization to the $24 \mathrm{hr}$ TMA, however, does not work for the stationary NS anisotropy. It is clear from (A1) that the stationary NS anisotropy $\xi_{z}^{\mathrm{GEO}}$ introduces no temporal variation to $I_{i, j}^{\mathrm{fit}}(t)$, while $\xi_{x}^{\mathrm{GEO}}$ and $\xi_{y}^{\mathrm{GEO}}$ cause a diurnal variation through terms of $\cos \omega t_{i}$ and $\sin \omega t_{i}$. If the NS anisotropy is stationary over a period longer than $24 \mathrm{hr}$, it is therefore eliminated by the normalization of $r_{i, j}^{\mathrm{obs}}(t)$ in equation (A5). We tested the normalization to the $24 \mathrm{hr}$ TMA, which resulted in a poor correlation between $\xi_{z}^{\mathrm{GEO}}$ and IMF sector polarity, even during a period when the GG component shows a good correlation.

To overcome this difficulty, we develop a new analysis method which is free from both atmospheric temperature effects and the normalization to the TMA. In this new method we obtain the anisotropy by best-fitting the model to the difference between the muon rate in each directional channel and the vertical channel for that detector. This subtraction efficiently eliminates the temperature effects, which are common to all directional channels at least as a first-order approximation (see Appendix B). We define the observed difference $\Delta I_{i, j}^{\mathrm{obs}}(t)$ as

$$
\Delta I_{i, j}^{\mathrm{obs}}(t)=r_{i, j}^{\mathrm{obs}}(t)-r_{i, 1}^{\mathrm{obs}}(t)
$$

where $r_{i, 1}^{\mathrm{obs}}(t)$ is the observed percent deviation for the vertical channel $(j=1)$ of the $i$ th detector. We fit to $\Delta I_{i, j}^{\mathrm{obs}}(t)$ the model function $\Delta I_{i, j}^{\mathrm{fit}}(t)$, given as

$\Delta I_{i, j}^{\mathrm{fit}}(t)=I_{0}^{\prime \prime}(t) \Delta P_{m i, j}+\xi_{x}^{\mathrm{GEO}}(t)\left(\Delta c_{1 i, j}^{1} \cos \omega t_{i}-\Delta s_{1 i, j}^{1} \sin \omega t_{i}\right)+\xi_{y}^{\mathrm{GEO}}(t)\left(\Delta s_{1 i, j}^{1} \cos \omega t_{i}+\Delta c_{1 i, j}^{1} \sin \omega t_{i}\right)+\xi_{z}^{\mathrm{GEO}}(t) \Delta c_{1 i, j}^{0}$,

where $\Delta P_{m i, j}, \Delta c_{1 i, j}^{1}, \Delta s_{1 i, j}^{1}$, and $\Delta c_{1 i, j}^{0}$ are calculated as

$$
\begin{array}{rr}
\Delta P_{m i, j}=\left(P_{m i, j}-P_{m i, 1}\right) / P_{m 1,1}, & \Delta c_{1 i, j}^{1}=c_{1 i, j}^{1}-c_{1 i, 1}^{1}, \\
\Delta s_{1 i, j}^{1}=s_{1 i, j}^{1}-s_{1 i, 1}^{1}, & \Delta c_{1 i, j}^{0}=c_{1 i, j}^{0}-c_{1 i, 1}^{0},
\end{array}
$$

with $P_{m i, 1}, c_{1 i, 1}^{1}, s_{1 i, 1}^{1}$ and $c_{1 i, 1}^{0}$ denoting the median primary rigidity and coupling coefficients for the vertical channel of the $i$ th detector. This best-fit gives no direct information on the GCR density $I_{0}(t)$, as the rigidity-independent density $\left[I_{0}^{\prime}(t)\right.$ in eq. (A2)] does not appear in equation (A8). To obtain $I_{0}(t)$, therefore, we first correct the observed vertical muon rate at Nagoya for atmospheric temperature effects by using high-altitude atmospheric data (see Appendix B). We then calculate $I_{0}(t)$ by subtracting best-fit $\boldsymbol{\xi}^{\mathrm{GEO}}(t)$ terms in equation (A1) from the temperature-corrected vertical muon rate. Black curves in Figures $8 b-8 e$ show the best-fit density and anisotropy derived by this method. It is clear that the spurious diurnal variation of the anisotropy arising from temperature effects is substantially removed. We also corrected the observed muon rate for temperature effects by using high-altitude atmospheric data available at the location closest to each muon detector in the GMDN (see Appendix B) and confirmed that these results were the same as those obtained by our new method. This shows that the new method succeeds in eliminating temperature effects. Note that we can only apply the correction in Appendix B for part of the entire period analyzed here because high-altitude atmospheric data were not available simultaneously for all muon detectors at many times.

In order to represent the NS anisotropy parallel to the Earth's rotation axis, a difference combination (GG) of directional count rates from the Nagoya telescope was developed. It is defined as

$$
r_{\mathrm{GG}}(t)=\left[r_{\mathrm{N} 2}(t)-r_{\mathrm{S} 2}(t)\right]+\left[r_{\mathrm{N} 2}(t)-r_{\mathrm{E} 2}(t)\right],
$$

where $r_{\mathrm{XX}}(t)$ is the percent deviation of the pressure-corrected muon rate in the directional channel XX (=N2, S2, E2) of the Nagoya muon detector from the average over each solar rotation period (Nagashima et al. 1972). The physical characteristics of the directional channels N2, S2, and E2 are listed in Table 1. The GG component is free from atmospheric temperature effects, which are common to all directional channels used in the right-hand side of equation (A10) and are removed by the subtractions (Sagisaka 1986). The GG component in CR 2043 is displayed in Figure 8f, where the fluctuation is filtered out by taking $5 \mathrm{hr}$ central moving averages. In this panel one can see a significant diurnal variation in the GG component superposed on the day-to-day variation (e.g., DOYs 150-155). This diurnal variation is due to the contribution from the diurnal anisotropy perpendicular to the Earth's rotation axis; i.e., $\xi_{x}^{\mathrm{GEO}}(t)$ and $\xi_{y}^{\mathrm{GEO}}(t)$ in equation (A1). The anisotropy component, when stationary and observed by a detector on the rotating Earth, produces a diurnal variation in which the muon rate varies as a sinusoidal function of local time. Since the amplitude and local time of maximum of this diurnal variation are both generally different in different directional channels, the diurnal variation remains in the GG component even after the subtractions in equation (A10). Owing to this contribution from the diurnal anisotropy, the GG component from a single muon detector at Nagoya cannot be used as an indicator of the NS anisotropy on an hourly basis. In many cases, it has been used only after averaging over a one day period (Mori \& Nagashima 1979; Laurenza \& Storini 2003). In contrast, by analyzing data from the muon detectors in the GMDN, we can derive the NS anisotropy separately from the diurnal anisotropy. The day-to-day variation shown by the black curve in Figure $8 e$ is in good agreement with that shown for the GG component in Figure $8 f$, implying that the new method successfully retains the NS anisotropy recorded by the GG component. Since this method gives us the NS anisotropy free from the diurnal variation, it enables us to examine the temporal variation within a day as well as the gradual day-to-day variation.

\section{A2. CORRELATION OF THE NS ANISOTROPY WITH THE IMF SECTOR POLARITY}

Figure 9 shows histograms of the GG component and the best-fit $\xi_{z}^{\mathrm{GEO}}$ in a period between 2006 March 14 and 2007 March 4. The upper two panels ( $a$ and $b$ ) display hourly values, while the lower two panels ( $c$ and $d$ ) show daily mean values. Figure $9 a$ shows that 


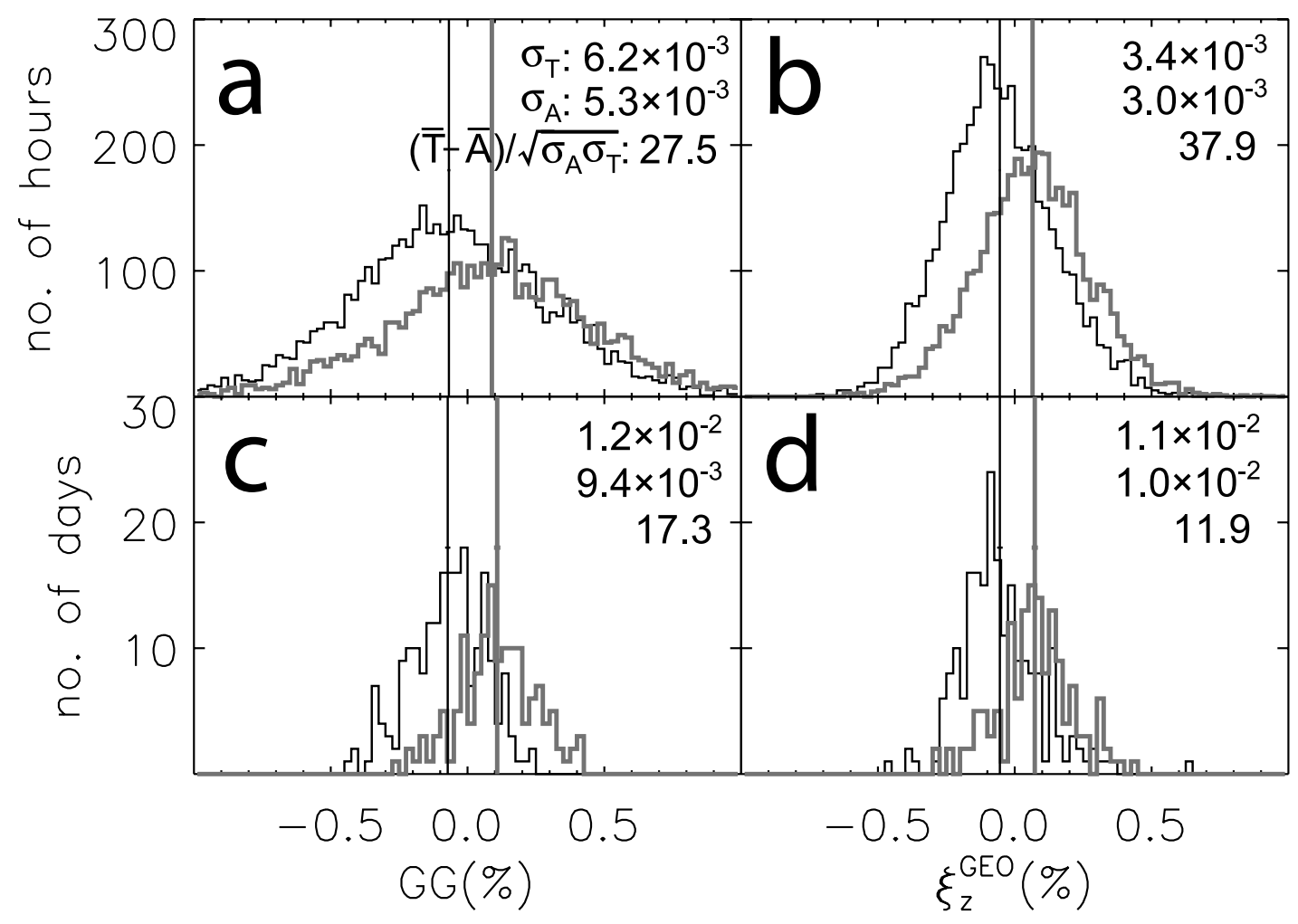

FIG. 9.-Correlation of the NS anisotropy with the IMF sector polarity over 13 solar rotations, CR 2041-CR 2053. The left two panels ( $a$ and $c$ ) display histograms of the GG component, while the right two panels ( $b$ and $d$ ) show histograms of $\xi_{z}^{\mathrm{GEO}}$ by the new analysis method. The gray and black traces in each panel show histograms in T and A sectors, respectively. The upper two panels are for hourly values, and the lower two panels are for daily mean values. The sector polarity (T/A) for the hourly value is defined by the GSE longitude of the hourly mean IMF. Average values in T and A sectors ( $\overline{\mathrm{T}}$ and $\overline{\mathrm{A}})$ are indicated by vertical lines. Standard deviations of T and A sectors $\left(\sigma_{\mathrm{T}}\right.$ and $\sigma_{\mathrm{A}}$ in $\left.\%\right)$ and statistical significances of T/A separation, defined as $(\overline{\mathrm{T}}-\overline{\mathrm{A}}) / \sqrt{\sigma_{\mathrm{T}} \sigma_{\mathrm{A}}}$, are also indicated in each panel.

the hourly values of the GG component have extremely large dispersion, partly due to the contribution from the diurnal anisotropy, as mentioned in the preceding subsection. Figures $9 b$ and $9 d$ display the histograms by the new analysis method. The dispersion in Figure $9 b$ is much smaller than that of the GG component in Figure $9 a$. The T/A separation in Figure $9 b$ is also significantly improved when compared with $\xi_{z}^{\mathrm{GEO}}$ by the normalization using the $24 \mathrm{hr}$ TMA.

\section{APPENDIX B}

\section{ATMOSPHERIC TEMPERATURE EFFECT ON THE MUON INTENSITY}

The fractional deviation of the muon intensity $I\left(x, \theta, E_{\mu}, t\right)$ from its average due to the atmospheric temperature effect at a time $t$, zenith angle $\theta$, atmospheric depth $x$, and muon threshold energy $E_{\mu}$ is given as

$$
\Delta I / I\left(x, \theta, E_{\mu}, t\right)=\int_{0}^{x} \alpha\left(x^{\prime}, \theta, E_{\mu}\right) \delta T\left(x^{\prime}, t\right) d x^{\prime},
$$

where $\delta T\left(x^{\prime}, t\right)$ is the temperature deviation from the average at an atmospheric depth $x^{\prime}$ and $\alpha\left(x^{\prime}, \theta, E_{\mu}\right)$ is the partial temperature coefficient (Sagisaka 1986). The muon intensity variation due to the temperature effect can be derived from this equation, when $\delta T\left(x^{\prime}, t\right)$ is available at various $x^{\prime}$. Accurate $\delta T\left(x^{\prime}, t\right)$ are seldom available for all $x^{\prime}$ and all muon detectors simultaneously. In this paper, we use an alternative correction for temperature effects. As described briefly in Appendix A, the negative temperature effect on the muon intensity measured with the surface-level detectors arises predominantly from the increase of muon decays arising from the increased path length caused by atmospheric expansion. A significant negative correlation is therefore expected between the altitude of the equi-pressure surface and the muon intensity; i.e., the muon intensity decreases with increasing this altitude due to atmospheric expansion.

Figure $10 a$ shows the percent deviation of the pressure-corrected hourly count rate of muons recorded in the vertical channel at Nagoya from its yearly mean in 2006, set to $100 \%$. The observed muon rate at Nagoya (gray curve) shows a seasonal variation, with a maximum in the northern hemisphere winter. We examine the temperature effect on the muon rate at Nagoya by analyzing high-altitude atmospheric data available from Shionomisaki (at geographical latitude and longitude of $33.5^{\circ} \mathrm{N}, 135.8^{\circ} \mathrm{E}$ ), located southwest of Nagoya. These radiosonde measurements have been continuously conducted by the Japan Meteorological Agency every $12 \mathrm{hr}$. The thin black curve in Figure $10 a$ displays the $100 \mathrm{hPa}$ altitude equi-pressure surface (henceforth called "the $100 \mathrm{hPa}$ altitude"). The anticorrelation between the muon rate and the $100 \mathrm{hPa}$ altitude is evident. The anticorrelation is confirmed in Figure $10 \mathrm{~b}$, showing the scatter plot between the two quantities, with a correlation coefficient of -0.947 and a regression coefficient of $-6.83 \% \mathrm{~km}^{-1}$. The muon rate 

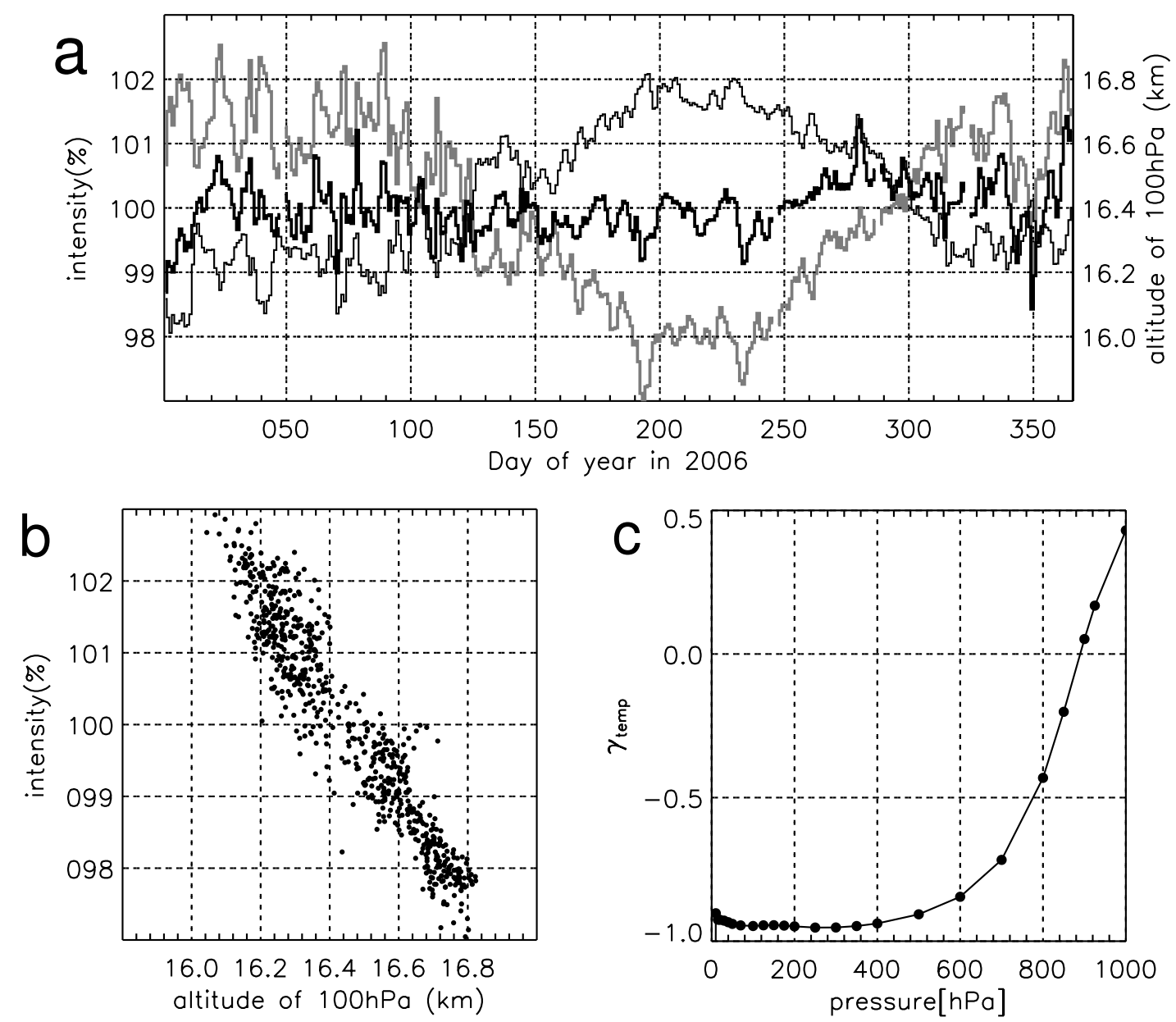

FIG. 10.-Atmospheric temperature effects on the pressure-corrected muon count rate. The top panel $(a)$ displays the observed vertical muon rates at Nagoya by a gray trace (with the left axis), the $100 \mathrm{hPa}$ altitude by the thin black trace (with the right axis), and the muon rate corrected for atmospheric temperature effects by the thick black trace (with the left axis), each as a function of time (DOY) in 2006. The bottom left panel (b) displays a scatter plot of the observed muon rate and the $100 \mathrm{hPa}$ altitude, while the bottom right panel $(c)$ shows the correlation coefficient between the observed muon rate and the altitude as a function of the atmospheric pressure of the equi-pressure surface.

corrected using the $100 \mathrm{hPa}$ altitude and this regression coefficient is shown by the thick black curve in Figure $10 a$. The seasonal variation in the gray curve is eliminated by this correction. Similar correlations are seen in Figure $10 \mathrm{c}$ for the $100-300 \mathrm{hPa}$ equi-pressure range. In this paper, we use the $100 \mathrm{hPa}$ altitude and the regression coefficient to correct the temperature effect on the hourly muon rate in each directional channel in the GMDN. For this correction, we calculate the hourly value of the $100 \mathrm{hPa}$ altitude by interpolating the data observed twice a day. We use the $100 \mathrm{hPa}$ altitude, also measured twice a day, at Porto Alegre $\left(30.0^{\circ} \mathrm{S}, 308.8^{\circ} \mathrm{E}\right), \operatorname{Hobart}\left(42.8^{\circ} \mathrm{S}, 147.5^{\circ} \mathrm{E}\right)$, and Kuwait $\left(29.2^{\circ} \mathrm{N}, 48.0^{\circ} \mathrm{E}\right)$ airports for corrections of the muon rates recorded by São Martinho, Hobart, and Kuwait University muon detectors, respectively. ${ }^{13}$ The distance between each muon detector and its associated meteorological station is $\sim 200 \mathrm{~km}$ for Shionomisaki, $\sim 260 \mathrm{~km}$ for Porto Alegre, $\sim 30 \mathrm{~km}$ for Hobart, and $\sim 10 \mathrm{~km}$ for Kuwait. The number of $100 \mathrm{hPa}$ altitude observations available, from twice daily measurements over the 13 rotation periods analyzed, is 693 from Shionomisaki, 576 from Hobart, 271 from Porto Alegre, and 492 from Kuwait. The correlation $\left(\gamma_{\text {temp }}\right)$ and regression $\left(\beta_{\text {temp }}\right)$ coefficients obtained for all directional channels in the GMDN are listed in the last two columns of Table 1 . Note that we cannot correct the GMDN data routinely with $\beta_{\text {temp }}$ in this table, because the $100 \mathrm{hPa}$ altitude data are not always available for all muon detectors. We could make such corrections for only 79 of the $\sim 350$ days analyzed here.

The correlations for Nagoya and Kuwait University are very significant, with $\gamma_{\text {temp }}$ less than -0.9 , while those for Hobart and São Martinho are less significant, with $\gamma_{\text {temp }}$ ranging from -0.773 to -0.735 . This is mainly due to the amplitude of the seasonal variation of the $100 \mathrm{hPa}$ altitude being different for the different locations. The correlation coefficient is high for Nagoya and Kuwait University, where the amplitudes are as large as 0.268 and $0.265 \mathrm{~km}$, respectively, while it is lower for Hobart and São Martinho, where the amplitudes are only 0.159 and $0.071 \mathrm{~km}$, respectively. It is noted in Table 1 that the $\beta_{\text {temp }}$ values are almost identical for all directional channels for the Nagoya and Kuwait University detectors, where the correlation is very significant. The fractional difference between the maximum and minimum $\beta_{\text {temp }}$ is only $14 \%$ for Nagoya and $20 \%$ for Kuwait University. It can also be seen for Nagoya that $\beta_{\text {temp }}$ is minimum for the vertical channel and gradually increases for more inclined channels. This confirms the expectation, on which we based the new analysis method for eliminating the temperature effect, that the effect is similar for all directional channels, at least as a firstorder approximation (see Appendix A).

13 The high-altitude atmospheric data are available at http://badc.nerc.ac.uk/home/. 


\section{REFERENCES}

Ahluwalia, H. S., \& Dorman, L. I. 1997, J. Geophys. Res., 102, 17433 Bieber, J. W., \& Chen, J. 1991, ApJ, 372, 301

Bieber, J. W., \& Evenson, P. 1998, Geophys. Res. Lett., 25, 2955

Chen, J., \& Bieber, J. W. 1993, ApJ, 405, 375

Hall, D. L., Duldig, M. L., \& Humble, J. E. 1996, Space Sci. Rev., 78, 401 1997, ApJ, 482, 1038

Jokipii, J. R., Kóta, J. 1982, Geophys. Res. Lett., 9, 656

Kóta, J. 1999, J. Geophys. Res., 104, 2499

Kóta, J., \& Jokipii, J. R. 2001, Adv. Space Res., 27, 607

Kuwabara, T., et al. 2004, Geophys. Res. Lett., 31, L19803

Laurenza, M., \& Storini, M. 2003, J. Geophys. Res., 108, 1069

Leerungnavarat, K., Ruffolo, D., \& Bieber, J. W. 2003, ApJ, 593, 587

Lin, Z., Bieber, J. W., \& Evenson, P. 1995, J. Geophys. Res., 100, 23543

Minnie, J., Bieber, J. W., Matthaeus, W. H., \& Burger, R. A. 2007, ApJ, 670, 1149

Mori, S., \& Nagashima, K. 1979, Planet. Space Sci., 27, 39
Munakata, K., et al. 2000, J. Geophys. Res., 105, 7457 2002, Adv. Space Res., 29, 1527

2005, Geophys. Res. Lett., 32, L03S04

Murakami, K., Nagashima, K., Sagisaka, S., Mishima, Y., \& Inoue, A. 1979, Nuovo Cim., 2C, 635

Nagashima, K., Fujimoto, K., Fujii, Z., Ueno, H., \& Kondo, I. 1972, Rep. Ionos. Space Res. Japan, 26, 31

Parker, E. N. 1965, Planet. Space Sci., 13, 9

Richardson, I. G. 2004, Space Sci. Rev., 111, 267

Richardson, I. G., Wibberenz, G., \& Cane, H. V. 1996, J. Geophys. Res., 101, 13483

Sabbah, I. 1999, Sol. Phys., 188, 401

Sagisaka, S. 1986, Nuovo Cim., 9C, 809

Swinson, D. B. 1969, J. Geophys. Res., 74, 5591 